\title{
MODELING OF WAVE RESONANCES IN LOW-CONTRAST PHOTONIC CRYSTALS*
}

\author{
DMITRI AGUEEV ${ }^{\dagger}$ AND DMITRY PELINOVSKY ${ }^{\dagger}$
}

\begin{abstract}
Coupled-mode equations are derived from Maxwell equations for modeling of lowcontrast cubic-lattice photonic crystals in three spatial dimensions. Coupled-mode equations describe resonantly interacting Bloch waves in stop bands of the photonic crystal. We study the linear boundary-value problem for stationary transmission of four counter-propagating and two oblique waves on the plane. Well-posedness of the boundary-value problem is proved by using the method of separation of variables and generalized Fourier series. For applications in photonic optics, we compute integral invariants for transmission, reflection, and diffraction of resonant waves.
\end{abstract}

Key words. photonic crystals, coupled-mode equations, wave resonances, stationary transmission boundary-value problems

AMS subject classifications. 35P10, 35P20, 35Q60, 78M35

DOI. $10.1137 / 040606053$

1. Introduction. Photonic band-gap crystals are periodic optical materials, the spectrum of which consists of bands separated by band gaps [13]. Linear periodic properties of the isotropic photonic crystals are modeled with the Maxwell equations

$$
\nabla^{2} \mathbf{E}-\frac{n^{2}}{c^{2}} \frac{\partial^{2} \mathbf{E}}{\partial t^{2}}=\nabla(\nabla \cdot \mathbf{E}), \quad \nabla \cdot\left(n^{2} \mathbf{E}\right)=0,
$$

where $n=n(\mathbf{x})$ is the periodic refractive index, $\mathbf{E}=\left(E_{x}, E_{y}, E_{z}\right)$ is the electric field vector, $\mathbf{x}=(x, y, z)$ is the physical space, $t$ is the time variable, $\nabla=\left(\partial_{x}, \partial_{y}, \partial_{z}\right)$ is the gradient vector, and $c$ is the speed of light. Components of the magnetic field vector are eliminated from the Maxwell equations (1.1) [13].

The Maxwell equations (1.1) in one dimension can be simplified for a linearly polarized light, such that $\mathbf{E}=(E, 0,0)$, where $E=E(z, t)$ and $n=n(z)$. The scalar component $E(z, t)$ solves the wave equation with the periodic speed variations

$$
\frac{\partial^{2} E}{\partial z^{2}}-\frac{n^{2}(z)}{c^{2}} \frac{\partial^{2} E}{\partial t^{2}}=0
$$

If the refractive index $n(z)$ is a periodic function with period $z_{0}$, the linear spectrum of the wave equation (1.2) reduces to the Mathieu equation for $E(z, t)=\psi(z) e^{-i \omega t}$, where $\omega$ is the eigenvalue and $\psi(z)$ is the eigenfunction of the spectral problem

$$
\psi^{\prime \prime}+\frac{\omega^{2}}{c^{2}} n^{2}(z) \psi=0 .
$$

According to the Floquet theory [12], solutions of the Mathieu equation (1.3) take the form $\psi(z)=\Psi(z) e^{i k(\omega) z}$, where $\Psi\left(z+z_{0}\right)=\Psi(z)$ and $k=k(\omega)$ is the propagation constant. For a general class of periodic potentials $n^{2}(z)$, there exist infinitely many

\footnotetext{
* Received by the editors March 31, 2004; accepted for publication (in revised form) August 20, 2004; published electronically April 14, 2005.

http://www.siam.org/journals/siap/65-4/60605.html

${ }^{\dagger}$ Department of Mathematics, McMaster University, 1280 Main Street West, Hamilton, ON, Canada, L8S 4K1 (agueevd@univmail.cis.mcmaster.ca, dmpeli@math.mcmaster.ca).
} 
intervals of $\omega$, called band gaps, where the propagation constant $k(\omega)$ is purely imaginary and the Bloch function $\psi(z)$ is unbounded in $z$. The band gaps are supported by the low-contrast photonic crystal with the refractive index $n(z)=n_{0}+\epsilon n_{1}(z)$, where $n_{0}$ is constant and $\epsilon$ is small parameter.

The linear Maxwell equations (1.1) in two and three dimensions can also be reduced to a spectral problem for $\mathbf{E}(\mathbf{x}, t)=\boldsymbol{\psi}(\mathbf{x}) e^{-i \omega t}$, where $\omega$ is the eigenvalue and $\boldsymbol{\psi}(\mathbf{x})$ is the eigenvector. When $n(\mathbf{x})$ is a periodic function in $x, y, z$ with periods $x_{0}$, $y_{0}, z_{0}$, respectively, the eigenvector $\boldsymbol{\psi}(\mathbf{x})$ satisfies the Floquet theorem [12] and has the form of the Bloch wave: $\boldsymbol{\psi}(\mathbf{x})=\mathbf{\Psi}(\mathbf{x}) e^{i\left(k_{x} x+k_{y} y+k_{z} z\right)}$, where $\mathbf{\Psi}(\mathbf{x})$ is periodic in $x, y$, and $z$ with periods $x_{0}, y_{0}$, and $z_{0}$, and $\omega=\omega\left(k_{x}, k_{y}, k_{z}\right)$. No band gaps exist in the linear spectrum for low-contrast photonic crystals. As a result, the bounded Bloch functions $\psi(\mathbf{x})$ may exist for any value of $\omega \in \mathbb{R}$. High-contrast photonic crystals may, however, exhibit band gaps for some configurations of the refractive index $n(\mathbf{x})$ [13]

Modeling of time-dependent responses of photonic crystals in three spatial dimensions can be computationally difficult in the framework of the Maxwell equations, especially if the nonlinear and nonlocal dispersive terms are taken into account. A more efficient method is based on reduction of Maxwell equations (1.1) to the coupledmode equations [23]. For instance, shock wave singularities may occur in the nonlinear Maxwell equations but they do not occur in the nonlinear coupled-mode equations [8]. Coupled-mode equations are typically derived in the first band gap of the Bragg resonance between two counter-propagating waves in one spatial dimension [20,21]. More complicated coupled-mode equations are considered for three-dimensional nonlinear photonic crystals $[1,2,3,6]$. Recent reviews $[4,5]$ also include classification of different resonances of Bloch waves in photonic crystals with quadratic nonlinearities.

In this paper, we classify wave resonances and coupled-mode equations for lowcontrast cubic-lattice photonic crystals in three spatial dimensions. Since low-contrast crystals do not support band gaps beyond one dimension [12, 13], resonances are considered in stop bands of the linear spectrum [10]. Stop bands occur between resonant counter-propagating waves, which could be coupled resonantly with other oblique Bloch waves. The number of resonant Bloch waves depends on the geometric configuration of the incident wave with respect to the cubic lattice. When the Maxwell equations are truncated with the perturbation series expansions, coupled-mode equations for the lowest-order Bragg resonances are derived and studied in bounded domains, subject to the radiation boundary conditions. The radiation boundary conditions describe transmission of the incident Bloch waves which generate resonantly reflected and diffracted Bloch waves in the photonic crystals.

We study here the linear coupled-mode equations for four counter-propagating and two oblique Bloch waves on the plane. It is not a priori clear why the stationary boundary-value problem with radiation boundary conditions is well posed, since it is specified by non-self-adjoint operators on the bounded domains. We prove, however, the well-posedness of the linear stationary problem by using separation of variables and generalized Fourier series [24]. Eigenfunction expansions and convergence of generalized Fourier series follow from the general theory [7]. As a result, we construct explicit analytical expressions for stationary transmission, reflection, and diffraction of resonant Bloch waves, which are used in modeling of the low-contrast photonic crystals.

Other applications of optical photonic structures include nonlinear phenomena, such as bistable stationary transmission and gap soliton propagation $[6,14,15,22]$. Very little is known about the persistence of such phenomena in two and three spatial 
dimensions, especially given that no band gap exists in low-contrast three-dimensional photonic structures. The coupled-mode equations can be generalized to include the weakly nonlinear (cubic) terms and to extend the time-dependent problems to the nonlinear coupled-mode equations [18, 19]. Well-posedness of the nonlinear stationary problems is beyond the scope of this manuscript, which only presents solutions of the linear stationary problems. Nevertheless, linear analysis opens the road to nonlinear analysis of the corresponding boundary-value problems.

The paper is organized as follows. Classification of resonances in low-contrast cubic-lattice crystals is given in section 2. Derivation of coupled-mode equations for lowest-order resonances is described in section 3. The linear stationary boundaryvalue problems for four counter-propagating and two oblique resonant Bloch waves are analyzed in section 4 . Section 5 concludes the paper. Appendix A gives derivation and explicit forms of the nonlinear coupled-mode equations with cubic (Kerr) nonlinearities.

2. Classification of resonances. When the optical material is homogeneous, such that $n(\mathbf{x})=n_{0}$ is constant, the linear spectrum of the Maxwell equations (1.1) is defined by the free transverse waves,

$$
\mathbf{E}(\mathbf{x}, t)=\mathbf{e}_{\mathbf{k}} e^{i(\mathbf{k} \cdot \mathbf{x}-\omega t)},
$$

where $\mathbf{e}_{\mathbf{k}}$ is the polarization vector, $\mathbf{k}=\left(k_{x}, k_{y}, k_{z}\right)$ is the wave vector, and $\omega=\omega(\mathbf{k})$ is the wave frequency. It follows from system (1.1) that

$$
\mathbf{k} \cdot \mathbf{e}_{\mathbf{k}}=0, \quad \omega^{2}=\frac{c^{2}}{n_{0}^{2}}\left(k_{x}^{2}+k_{y}^{2}+k_{z}^{2}\right)
$$

For each wave vector $\mathbf{k}$ there exist two independent polarizations $\mathbf{e}_{\mathbf{k}}^{(1)}$ and $\mathbf{e}_{\mathbf{k}}^{(2)}$ such that $\mathbf{e}_{\mathbf{k}}^{(1)} \cdot \mathbf{e}_{\mathbf{k}}^{(2)}=0$. This degeneracy in the polarization vector is neglected here by the assumption that the incident wave is linearly polarized.

When the optical material is periodic such that $n\left(\mathbf{x}+\mathbf{x}_{0}\right)=n\left(\mathbf{x}_{0}\right)$, the linear spectrum of the Maxwell equations (1.1) is defined by the Bloch waves:

$$
\mathbf{E}(\mathbf{x}, t)=\mathbf{\Psi}(\mathbf{x}) e^{i(\mathbf{k} \cdot \mathbf{x}-\omega t)},
$$

where $\mathbf{\Psi}\left(\mathrm{x}+\mathrm{x}_{0}\right)=\mathbf{\Psi}(\mathrm{x})$ is the periodic envelope, $\mathbf{k}=\left(k_{x}, k_{y}, k_{z}\right)$ is the wave vector, and $\omega=\omega(\mathbf{k})$ is the wave frequency. Existence of the Bloch waves (2.3) for the Maxwell equations (1.1) is proved in [12]. The geometric configuration of the photonic crystal is defined by the fundamental (linearly independent) lattice vectors $\mathbf{x}_{1,2,3}$ and fundamental reciprocal lattice vectors $\mathbf{k}_{1,2,3}$ such that $\mathbf{k}_{i} \cdot \mathbf{x}_{j}=2 \pi \delta_{i, j}$, where $1 \leq i$, $j \leq 3$ (see [10]). Therefore, the linear refractive index $n(\mathbf{x})$ can be expanded into a triple Fourier series:

$$
n(\mathbf{x})=n_{0} \sum_{(n, m, l) \in \mathbb{Z}^{3}} \alpha_{n, m, l} e^{i\left(n \mathbf{k}_{1}+m \mathbf{k}_{2}+l \mathbf{k}_{3}\right) \cdot \mathbf{x}},
$$

where the factor $n_{0}$ is included for convenience. If $n_{0}$ is the mean value of $n(\mathbf{x})$, then $\alpha_{0,0,0}=1$. Let the wave vector $\mathbf{k}$ in the incident Bloch wave (2.3) be chosen as $\mathbf{k}=\mathbf{k}_{\mathrm{in}}$. The incident wave vector $\mathbf{k}_{\mathrm{in}}$ is expanded in terms of the lattice vectors:

$$
\mathbf{k}_{\mathrm{in}}=\frac{1}{2}\left(p \mathbf{k}_{1}+q \mathbf{k}_{2}+r \mathbf{k}_{3}\right),
$$


where $(p, q, r) \in \mathbb{R}^{3}$ are parameters. The Bloch wave (2.3) is represented by triple Fourier series for $\mathbf{\Psi}(\mathbf{x})$, such that $\mathbf{E}(\mathbf{x}, t)$ consists of an infinite superposition of free transverse waves with the wave vectors $\mathbf{k}_{\text {out }}^{(n, m, l)}$ :

$$
\mathbf{k}_{\text {out }}^{(n, m, l)}=\mathbf{k}_{\text {in }}+n \mathbf{k}_{1}+m \mathbf{k}_{2}+l \mathbf{k}_{3}, \quad(n, m, l) \in \mathbb{Z}^{3} .
$$

The wave vector $\mathbf{k}_{\text {out }}^{(n, m, l)}$ with a nonempty triple $(n, m, l)$ is said to be resonant with the wave vector $\mathbf{k}_{\text {in }}$ if $\left|\mathbf{k}_{\text {out }}^{(n, m, l)}\right|=\left|\mathbf{k}_{\text {in }}\right|$ such that $\left|\omega\left(\mathbf{k}_{\text {out }}^{(n, m, l)}\right)\right|=\left|\omega\left(\mathbf{k}_{\text {in }}\right)\right|$.

We consider here a simple cubic crystal, where the fundamental lattice vectors and reciprocal lattice vectors are all orthogonal [10]:

$$
\mathbf{x}_{1,2,3}=a \mathbf{e}_{1,2,3}, \quad \mathbf{k}_{1,2,3}=k_{0} \mathbf{e}_{1,2,3}, \quad k_{0}=\frac{2 \pi}{a},
$$

where $\mathbf{e}_{1,2,3}$ are unit vectors in $\mathbb{R}^{3}$. The coordinate axes $(x, y, z)$ are oriented along the axes of the simple cubic crystal, while the incident wave vector $\mathbf{k}_{\text {in }}$ is directed according to the spherical angles $(\theta, \varphi)$ as follows:

$$
\mathbf{k}_{\mathrm{in}}=k(\sin \theta \cos \varphi, \sin \theta \sin \varphi, \cos \theta), \quad k \in \mathbb{R}, 0 \leq \theta \leq \pi, 0 \leq \varphi \leq 2 \pi,
$$

where $k=\left|\mathbf{k}_{\text {in }}\right|$. When $\theta=0$, the wave vector $\mathbf{k}_{\text {in }}$ is perpendicular to the $(x, y)$ crystal plane. For the simple cubic crystal, the set of resonant Bloch waves is given by the set of triples

$$
\mathcal{S}=\left\{(n, m, l) \in \mathbb{Z}^{3}: n(n+p)+m(m+q)+l(l+r)=0\right\},
$$

where

$$
p=\frac{2 k}{k_{0}} \sin \theta \cos \varphi, \quad q=\frac{2 k}{k_{0}} \sin \theta \sin \varphi, \quad r=\frac{2 k}{k_{0}} \cos \theta .
$$

The set $\mathcal{S}$ always has a zero solution: $(n, m, l)=(0,0,0)$. When $(p, q, r) \in \mathbb{Z}^{3}$ and $|p|+|q|+|r| \neq 0$, the set $\mathcal{S}$ has at least one nonzero solution: $(n, m, l)=(-p,-q,-r)$. The set $\mathcal{S}$ is also bounded, since $(n, m, l)$ are integer solutions inside the sphere:

$$
\left(n+\frac{p}{2}\right)^{2}+\left(m+\frac{q}{2}\right)^{2}+\left(l+\frac{r}{2}\right)^{2}=\left(\frac{k}{k_{0}}\right)^{2}<\infty .
$$

When $(p, q, r) \in \mathbb{Z}^{3}$, resonant triples $(n, m, l)$ can all be classified analytically. However, when $(p, q, r) \notin \mathbb{Z}^{3}$, additional resonant triples may also exist. In solid state physics [10], a geometric solution for the resonant triples $(n, m, l)$ is constructed from the condition that the vector $\mathbf{G}^{(n, m, l)}=\mathbf{k}_{\text {out }}^{(n, m, l)}-\mathbf{k}_{\text {in }}$ lies on the edge of sectors of the reciprocal lattice. Here we review particular resonant sets $\mathcal{S}$ for integer and noninteger values of $(p, q, r)$.

2.1. A family of one-dimensional resonances. The one-dimensional Bragg resonance occurs when the incident wave is coupled with the counter-propagating reflected wave such that the set $\mathcal{S}$ has at least one nonzero solution: $(n, m, l)=$ $(0,0,-r)$, where $r \in \mathbb{Z}_{+}$. The values of $p$ and $q$ are not defined for the Bragg resonance when $n=m=0$. As a result, spherical angles $\theta$ and $\varphi$ in the parametrization (2.8) are arbitrary, while the wave number $k$ satisfies the Bragg resonance condition [10]:

$$
r k_{0}=2 k \cos \theta
$$


such that $r \lambda=2 a \cos \theta$, where $\lambda$ is the wavelength. The one-dimensional Bragg resonance is generalized in three dimensions for $p=q=0$ and $r \in \mathbb{Z}_{+}$, when the geometric configuration for the Bragg resonance (2.12) is fixed at the specific value $\theta=0$, and

$$
\mathbf{k}_{\mathrm{in}}=\frac{\pi}{a}(0,0, r), \quad \mathbf{k}_{\mathrm{out}}^{(0,0,-r)}=\frac{\pi}{a}(0,0,-r) .
$$

The incident wave is directed to the $z$-axis of the cubic lattice crystal, and the wavelength is $\lambda=2 a / r$. The family of Bragg resonances with $p=q=0$ and $r \in \mathbb{Z}_{+}$ may include not only the two counter-propagating waves (2.13) but also other Bloch waves in three-dimensional photonic crystals. The lowest-order resonant sets $\mathcal{S}$ for $p=q=0$ and $r \in \mathbb{Z}_{+}$are listed below:

$$
\begin{aligned}
r=1: \mathcal{S}= & \{(0,0,0),(0,0,-1)\} \\
r=2: \mathcal{S}= & \{(0,0,0),(1,0,-1),(-1,0,-1),(0,1,-1),(0,-1,-1),(0,0,-2)\} \\
r=3: \mathcal{S}= & \{(0,0,0),(1,1,-1),(-1,1,-1),(1,-1,-1),(-1,-1,-1) \\
& (1,1,-2),(-1,1,-2),(1,-1,-2),(-1,-1,-2),(0,0,-3)\}
\end{aligned}
$$

The dimension of $\mathcal{S}$ depends on the total number of all possible integer solutions for $(n, m, l)$. The sets $\mathcal{S}$ for higher-order resonances with $r \in \mathbb{Z}_{+}$can be found algorithmically, with symbolic computing software.

2.2. A family of two-dimensional resonances. Two-dimensional Bragg resonances occur when the incident wave vector $\mathbf{k}_{\text {in }}$ is resonant to the counter-propagating reflected wave vector $\mathbf{k}_{\text {out }}^{(-p,-q, 0)}$, as well as to two other diffracted wave vectors $\mathbf{k}_{\text {out }}^{(0,-q, 0)}$ and $\mathbf{k}_{\text {out }}^{(-p, 0,0)}$, where $(p, q) \in \mathbb{Z}_{+}^{2}$. The value of $r$ is not defined for the two-dimensional resonance, such that the angle $\theta$ in the parametrization (2.8) is arbitrary, while $k$ and $\varphi$ satisfy the resonance conditions

$$
\varphi=\arctan \left(\frac{q}{p}\right), \quad \sqrt{p^{2}+q^{2}} k_{0}=2 k \sin \theta .
$$

The two-dimensional Bragg resonances are generalized in three dimensions for $(p, q) \in$ $\mathbb{Z}_{+}^{2}$ and $r=0$, when the geometric configuration for the Bragg resonance (2.14) is fixed at the specific value $\theta=\frac{\pi}{2}$, and

$$
\begin{aligned}
\mathbf{k}_{\text {in }} & =\frac{\pi}{a}(p, q, 0), & \mathbf{k}_{\text {out }}^{(-p,-q, 0)} & =\frac{\pi}{a}(-p,-q, 0), \\
\mathbf{k}_{\text {out }}^{(0,-q, 0)} & =\frac{\pi}{a}(p,-q, 0), & \mathbf{k}_{\text {out }}^{(-p, 0,0)} & =\frac{\pi}{a}(-p, q, 0) .
\end{aligned}
$$

The incident wave $\mathbf{k}_{\text {in }}$ is directed along the diagonal of the $(p x, q y)$-cell of the cubic lattice crystal, and the wavelength is $\lambda=2 a / \sqrt{p^{2}+q^{2}}$.

The families of Bragg resonances with $(p, q) \in \mathbb{Z}_{+}^{2}$ and $r=0$ may include not only the four resonant waves (2.15) but also other Bloch waves in three-dimensional photonic crystals. The lowest-order resonant sets $\mathcal{S}$ for $(p, q) \in \mathbb{Z}_{+}^{2}$ and $r=0$ are listed below:

$$
\begin{aligned}
p=1, q=1: \mathcal{S}= & \{(0,0,0),(-1,0,0),(0,-1,0),(-1,-1,0)\}, \\
p=2, q=1: \mathcal{S}= & \{(0,0,0),(0,-1,0),(-1,0,1),(-1,0,-1),(-1,-1,1),(-1,-1,-1), \\
& (-2,0,0),(-2,-1,0)\},
\end{aligned}
$$




$$
\begin{aligned}
p=2, q=2: \mathcal{S}=\{ & (0,0,0),(0,-1,1),(0,-1,-1),(0,-2,0),(-1,0,1),(-1,0,-1), \\
& (-1,-2,1),(-1,-2,-1),(-2,0,0),(-2,-1,1),(-2,-1,-1), \\
& (-2,-2,0)\} .
\end{aligned}
$$

2.3. Two-dimensional resonances of oblique waves. The resonant set $\mathcal{S}$ can be nonempty for $(p, q, r) \notin \mathbb{Z}^{3}$, which correspond to oblique Bloch waves. For instance, two oblique waves can be resonant on the $(x, y)$-plane if

$$
\mathbf{k}_{\mathrm{in}}=\frac{\pi}{a}(p, q, 0), \quad \mathbf{k}_{\mathrm{out}}^{(n, m, 0)}=\frac{\pi}{a}(p+2 n, q+2 m, 0),
$$

where $(n, m) \in \mathbb{Z}^{2}$ are arbitrary and $(p, q) \in \mathbb{R}^{2}$ are taken on the straight line:

$$
n p+m q=-\left(n^{2}+m^{2}\right) .
$$

Similarly, three oblique waves can be resonant on the $(x, y)$-plane if

$$
\begin{aligned}
\mathbf{k}_{\text {in }} & =\frac{\pi}{a}(p, q, 0), \\
\mathbf{k}_{\text {out }}^{\left(n_{1}, m_{1}, 0\right)} & =\frac{\pi}{a}\left(p+2 n_{1}, q+2 m_{1}, 0\right), \\
\mathbf{k}_{\text {out }}^{\left(n_{2}, m_{2}, 0\right)} & =\frac{\pi}{a}\left(p+2 n_{2}, q+2 m_{2}, 0\right),
\end{aligned}
$$

where $\left(n_{1}, m_{1}\right) \in \mathbb{Z}^{2}$ and $\left(n_{2}, m_{2}\right) \in \mathbb{Z}^{2}$ are arbitrary subject to the constraint $m_{1} n_{2} \neq$ $m_{2} n_{1}$, while $(p, q)$ take rational values

$$
p=\frac{m_{1}\left(n_{2}^{2}+m_{2}^{2}\right)-m_{2}\left(n_{1}^{2}+m_{1}^{2}\right)}{m_{2} n_{1}-m_{1} n_{2}}, \quad q=\frac{n_{1}\left(n_{2}^{2}+m_{2}^{2}\right)-n_{2}\left(n_{1}^{2}+m_{1}^{2}\right)}{n_{2} m_{1}-n_{1} m_{2}} .
$$

In the general case, two oblique waves (2.16) or three oblique waves (2.18) may have resonances with other Bloch waves in three-dimensional photonic crystals.

2.4. A family of three-dimensional resonances. When $(p, q, r) \in \mathbb{Z}_{+}^{3}$, the resonant sets $\mathcal{S}$ include eight coupled waves for fully three-dimensional Bragg resonance:

$$
\begin{aligned}
& \mathbf{k}_{\mathrm{in}}=\frac{\pi}{a}(p, q, r), \quad \mathbf{k}_{\mathrm{out}}^{(-p,-q,-r)}=\frac{\pi}{a}(-p,-q,-r), \\
& \mathbf{k}_{\text {out }}^{(-p, 0,0)}=\frac{\pi}{a}(-p, q, r), \quad \mathbf{k}_{\text {out }}^{(0,-q, 0)}=\frac{\pi}{a}(p,-q, r), \\
& \mathbf{k}_{\text {out }}^{(0,0,-r)}=\frac{\pi}{a}(p, q,-r), \quad \mathbf{k}_{\text {out }}^{(-p,-q, 0)}=\frac{\pi}{a}(-p,-q, r), \\
& \mathbf{k}_{\text {out }}^{(-p, 0,-r)}=\frac{\pi}{a}(-p, q,-r), \quad \mathbf{k}_{\text {out }}^{(0,-q,-r)}=\frac{\pi}{a}(p,-q,-r) .
\end{aligned}
$$

The resonance condition for the three-dimensional Bragg resonance takes the form

$$
\varphi=\arctan \left(\frac{q}{p}\right), \quad \theta=\arctan \left(\frac{\sqrt{p^{2}+q^{2}}}{r}\right), \quad \sqrt{p^{2}+q^{2}+r^{2}} k_{0}=2 k .
$$

The incident wave $\mathbf{k}_{\mathrm{in}}$ is directed along the diagonal of the ( $\left.p x, q y, r z\right)$-cell of the cubic lattice crystal, and the wavelength is $\lambda=2 a / \sqrt{p^{2}+q^{2}+r^{2}}$. The eight waves (2.20) can be coupled with some other resonant waves such that $\operatorname{dim}(\mathcal{S}) \geq 8$ for $(p, q, r) \in \mathbb{Z}_{+}^{3}$. For instance, $\operatorname{dim}(\mathcal{S})=8$ for $(p, q, r)=(1,1,1)$ and $(p, q, r)=(2,1,1)$, but $\operatorname{dim}(\mathcal{S})=10$ for $(p, q, r)=(2,2,1)$ and $\operatorname{dim}(\mathcal{S})=16$ for $(p, q, r)=(3,2,1)$. 
3. Derivation of coupled-mode equations. The dispersion surface $\omega=\omega(\mathbf{k})$ for the Bloch waves (2.3) in the periodic photonic crystal is defined by the profile of the refractive index $n(\mathbf{x})$. We shall consider the asymptotic approximation of the dispersion surface $\omega=\omega(\mathbf{k})$ in the limit when the photonic crystal is low-contrast, such that the refractive index $n(\mathbf{x})$ is given by

$$
n(\mathbf{x})=n_{0}+\epsilon n_{1}(\mathbf{x}),
$$

where $n_{0}$ is a constant and $\epsilon$ is small parameter. It is proved in [12] that the Bloch waves (2.3) are smooth functions of $\epsilon$, such that the asymptotic solution of the Maxwell equations (1.1) as $\epsilon \rightarrow 0$ takes the form of the perturbation series expansions:

$$
\mathbf{E}(\mathbf{x}, t)=\mathbf{E}_{0}(\mathbf{x}, t)+\epsilon \mathbf{E}_{1}(\mathbf{x}, t)+\mathrm{O}\left(\epsilon^{2}\right) .
$$

The leading-order term $\mathbf{E}_{0}(\mathbf{x}, t)$ consists of free transverse waves (2.1) with wave vectors $\mathbf{k}_{\text {out }}^{(n, m, l)}$, given by (2.6), such that the asymptotic form (3.2) represents the Bloch wave $(2.3)$ as $\epsilon \neq 0$.

Coupled-mode equations are derived by separating resonant free waves from nonresonant free waves in the Bloch wave (2.3), where the resonant set $\mathcal{S}$ with $N=\operatorname{dim}(\mathcal{S})<\infty$ is defined by (2.9). Let $\mathbf{E}_{0}(\mathbf{x}, t)$ be a linear superposition of $N$ resonant waves with wave vectors $\mathbf{k}_{j}$ at the same frequency $\omega$ :

$$
\mathbf{E}_{0}(\mathbf{x}, t)=\sum_{j=1}^{N} A_{j}(\mathbf{X}, T) \mathbf{e}_{\mathbf{k}_{j}} e^{i\left(\mathbf{k}_{j} \mathbf{x}-\omega t\right)}, \quad \mathbf{X}=\frac{\epsilon \mathbf{x}}{k}, T=\frac{\epsilon t}{\omega},
$$

where $\omega$ and $\mathbf{k}_{j}$ are related by the same dispersion equation $(2.2), A_{j}(\mathbf{X}, T)$ is the envelope amplitude of the $j$ th resonant wave $(2.1)$, and $(\mathbf{X}, T)$ are slow variables. The slow variables represent a deformation of the dispersion surface $\omega=\omega\left(\mathbf{k}_{j}\right)$ due to the low-contrast periodic photonic crystal. The degeneracy in the polarization vector is neglected by the assumption that the incident wave is linearly polarized with the polarization vector $\mathbf{e}_{\text {in }}=\mathbf{e}_{\mathbf{k}_{\text {in }}}$. The triple Fourier series (2.4) for the cubic-lattice crystal (2.7) is simplified as follows:

$$
n_{1}(\mathbf{x})=n_{0} \sum_{(n, m, l) \in \mathbb{Z}^{3}} \alpha_{n, m, l} e^{i k_{0}(n x+m y+l z)},
$$

where $\alpha_{0,0,0}=0$. The Fourier coefficients $\alpha_{n, m, l}$ satisfy the constraints

$$
\alpha_{n, m, l}=\bar{\alpha}_{-n,-m,-l},
$$

due to the reality of $n_{1}(\mathbf{x})$;

$$
\alpha_{n, m, l}=\alpha_{m, n, l}=\alpha_{n, l, m}=\alpha_{l, m, n},
$$

due to the crystal isotropy in the directions of $x, y, z$-axes; and

$$
\alpha_{-n, m, l}=\alpha_{n, m, l}, \quad \alpha_{n,-m, l}=\alpha_{n, m, l}, \quad \alpha_{n, m,-l}=\alpha_{n, m, l},
$$

due to the crystal symmetry with respect to the origin $(0,0,0)$. (The latter property can be achieved by a simple shift of $(x, y, z)$.) It follows from constraints (3.5) and (3.7) that all coefficients $\alpha_{n, m, l}$ for $(n, m, l) \in \mathbb{Z}^{3}$ are real-valued. 
It follows from (1.1), (3.1), and (3.2) that the first-order correction term $\mathbf{E}_{1}(\mathbf{x}, t)$ solves the nonhomogeneous linear problem

$$
\begin{aligned}
\nabla^{2} \mathbf{E}_{1}-\frac{n_{0}^{2}}{c^{2}} \frac{\partial^{2} \mathbf{E}_{1}}{\partial t^{2}}=2 & \frac{n_{0}^{2} \omega}{c^{2}} \frac{\partial^{2} \mathbf{E}_{0}}{\partial T \partial t}-2 k\left(\nabla \cdot \nabla_{X}\right) \mathbf{E}_{0} \\
& +\frac{2 n_{0} n_{1}(\mathbf{x})}{c^{2}} \frac{\partial^{2} \mathbf{E}_{0}}{\partial t^{2}}+\frac{2}{n_{0}} \nabla\left(\nabla n_{1} \cdot \mathbf{E}_{0}\right)
\end{aligned}
$$

where $\nabla_{X}=\left(\partial_{X}, \partial_{Y}, \partial_{Z}\right)$ and the second equation of (1.1) has been used. The righthand side of the nonhomogeneous equation (3.8) has resonant terms, which are parallel to the free-wave resonant solutions of the homogeneous problem. The resonant terms lead to the secular growth of $\mathbf{E}_{1}(\mathbf{x}, t)$ in $t$ unless they are identically zero. The latter conditions define the coupled-mode equations for amplitudes $A_{j}(\mathbf{X}, T), j=1, \ldots, N$, in the general form

$$
i\left(\frac{\partial A_{j}}{\partial T}+\left(\frac{\mathbf{k}_{j}}{k} \cdot \nabla_{X}\right) A_{j}\right)+\sum_{k \neq j} \hat{\alpha}_{j, k} A_{k}=0, \quad j=1, \ldots, N,
$$

where the elements $\left\{\hat{\alpha}_{j, k}\right\}_{1 \leq j, k \leq N}$ are related to the Fourier coefficients of the resonant waves $\left\{\alpha_{n, m, l}\right\}_{(n, m, l) \in \mathcal{S}}$. The explicit forms of the coupled-mode equations (3.9) are given for two and four counter-propagating and two oblique resonant Bloch waves.

3.1. Coupled-mode equations for two counter-propagating waves. The lowest-order Bragg resonance for two counter-propagating waves (2.13) occurs for $r=1$, when

$$
\mathbf{k}_{1}=\frac{\pi}{a}(0,0,1), \quad \mathbf{k}_{2}=\frac{\pi}{a}(0,0,-1) .
$$

Let $A_{1}=A_{+}(Z, T)$ and $A_{2}=A_{-}(Z, T)$ be the amplitudes of the right (forward) and left (backward) propagating waves, respectively. The envelope amplitudes are not modulated across the $(X, Y)$-plane, since the coupled-mode equations for $A_{ \pm}$are essentially one-dimensional. The polarization vectors are chosen in the $x$-direction such that $\mathbf{e}_{\mathbf{k}_{1}}=\mathbf{e}_{\mathbf{k}_{2}}=(1,0,0)$ and $\mathbf{E}_{0}=\left(E_{0, x}(z, Z, T) e^{-i \omega t}, 0,0\right)$. The nonhomogeneous equation (3.8) at the $x$-component of the solution $\mathbf{E}_{1}$ at $e^{-i \omega t}$ takes the form

$$
\begin{aligned}
\nabla^{2} E_{1, x}+k^{2} E_{1, x}= & -2 i k^{2} \frac{\partial}{\partial T} E_{0, x}-2 k \frac{\partial^{2}}{\partial Z \partial z} E_{0, x} \\
& -\frac{2 k^{2} n_{1}(\mathbf{x})}{n_{0}} E_{0, x}+\frac{2}{n_{0}} \frac{\partial^{2} n_{1}(\mathbf{x})}{\partial x^{2}} E_{0, x} .
\end{aligned}
$$

By removing the resonant terms at $e^{ \pm i k z}$, the coupled-mode equations for amplitudes $A_{ \pm}(Z, T)$ take the form

$$
\begin{aligned}
& i\left(\frac{\partial A_{+}}{\partial T}+\frac{\partial A_{+}}{\partial Z}\right)+\alpha A_{-}=0 \\
& i\left(\frac{\partial A_{-}}{\partial T}-\frac{\partial A_{-}}{\partial Z}\right)+\alpha A_{+}=0
\end{aligned}
$$

where $\alpha=\alpha_{0,0,1}=\alpha_{0,0,-1}$. The coupled-mode equations (3.12)-(3.13) can be defined on the interval $0 \leq Z \leq L_{z}$ for $T \geq 0$, where the end points at $Z=0$ and $Z=L_{z}$ are the left and right $(x, y)$-planes, which cut a slice of the photonic crystal. The linear system (3.12)-(3.13) is reviewed in [23]. The nonlinear coupled-mode equations are derived in $[6,22]$ and analyzed recently in $[8,14,15]$. 
3.2. Coupled-mode equations for four counter-propagating waves. The lowest-order resonance for four counter-propagating waves (2.15) occurs for $p=q=1$, when

$$
\mathbf{k}_{1}=\frac{\pi}{a}(1,1,0), \quad \mathbf{k}_{2}=\frac{\pi}{a}(1,-1,0), \quad \mathbf{k}_{3}=\frac{\pi}{a}(-1,1,0), \quad \mathbf{k}_{4}=\frac{\pi}{a}(-1,-1,0) .
$$

Let $A_{1}=A_{+}(X, Y, T)$ and $A_{4}=A_{-}(X, Y, T)$ be the amplitudes of the counterpropagating waves along the main diagonal of the $(x, y)$ plane, while $A_{2}=B_{+}(X, Y, T)$ and $A_{3}=B_{-}(X, Y, T)$ are the amplitudes of the counter-propagating waves along the antidiagonal of the $(x, y)$-plane. The envelope amplitudes are not modulated in the $Z$-direction, since the coupled-mode equations for $A_{ \pm}$and $B_{ \pm}$are essentially twodimensional. The polarization vectors are chosen in the $z$-direction such that $\mathbf{e}_{\mathbf{k}_{j}}=$ $(0,0,1), 1 \leq j \leq 4$, and $\mathbf{E}_{0}=\left(0,0, E_{0, z}(x, y, X, Y, T) e^{-i \omega t}\right)$. The nonhomogeneous equation (3.8) at the $z$-component of the solution $\mathbf{E}_{1}$ at $e^{-i \omega t}$ takes the form

$$
\begin{aligned}
\nabla^{2} E_{1, z}+k^{2} E_{1, z}= & -2 i k^{2} \frac{\partial}{\partial T} E_{0, z}-2 k \frac{\partial^{2}}{\partial X \partial x} E_{0, z}-2 k \frac{\partial^{2}}{\partial Y \partial y} E_{0, z} \\
& -\frac{2 k^{2} n_{1}(\mathbf{x})}{n_{0}} E_{0, z}+\frac{2}{n_{0}} \frac{\partial^{2} n_{1}(\mathbf{x})}{\partial z^{2}} E_{0, z} .
\end{aligned}
$$

By removing the resonant terms at $e^{\frac{i}{\sqrt{2}}( \pm k x \pm k y)}$, the coupled-mode equations for amplitudes $A_{ \pm}(X, Y, T)$ and $B_{ \pm}(X, Y, T)$ take the form

$$
\begin{aligned}
& i\left(\frac{\partial A_{+}}{\partial T}+\frac{\partial A_{+}}{\partial X}+\frac{\partial A_{+}}{\partial Y}\right)+\alpha A_{-}+\beta\left(B_{+}+B_{-}\right)=0, \\
& i\left(\frac{\partial A_{-}}{\partial T}-\frac{\partial A_{-}}{\partial X}-\frac{\partial A_{-}}{\partial Y}\right)+\alpha A_{+}+\beta\left(B_{+}+B_{-}\right)=0, \\
& i\left(\frac{\partial B_{+}}{\partial T}+\frac{\partial B_{+}}{\partial X}-\frac{\partial B_{+}}{\partial Y}\right)+\beta\left(A_{+}+A_{-}\right)+\alpha B_{-}=0, \\
& i\left(\frac{\partial B_{-}}{\partial T}-\frac{\partial B_{-}}{\partial X}+\frac{\partial B_{-}}{\partial Y}\right)+\beta\left(A_{+}+A_{-}\right)+\alpha B_{+}=0,
\end{aligned}
$$

where $\alpha=\alpha_{1,1,0}=\alpha_{-1,-1,0}=\alpha_{1,-1,0}=\alpha_{-1,1,0}$ and $\beta=\alpha_{0,1,0}=\alpha_{1,0,0}=\alpha_{0,-1,0}=$ $\alpha_{-1,0,0}$. The coupled-mode equations (3.16)-(3.19) can be defined in the domain $(X, Y) \in \mathcal{D}$ and $T \geq 0$, where $\mathcal{D}$ is a domain on the $(x, y)$-plane of the photonic crystal. The system has not been previously studied in literature, to the best of our knowledge.

3.3. Coupled-mode equations for two oblique waves. Two oblique resonant waves on the $(x, y)$-plane are defined by the resonant wave vectors $(2.16)$ under the constraint (2.17). Assuming that $\mathbf{e}_{1}=\mathbf{e}_{2}=(0,0,1)$, the Maxwell equations can be reduced to the same form (3.15), where the resonant terms are eliminated at the wave vectors $\mathbf{k}_{1}=\mathbf{k}_{\text {in }}$ and $\mathbf{k}_{2}=\mathbf{k}_{\text {out }}^{(n, m, 0)}$. The coupled-mode equations for amplitudes $A_{1,2}(X, Y, T)$ take the form

$$
\begin{aligned}
& i\left(\frac{\partial A_{1}}{\partial T}+\frac{p}{\sqrt{p^{2}+q^{2}}} \frac{\partial A_{1}}{\partial X}+\frac{q}{\sqrt{p^{2}+q^{2}}} \frac{\partial A_{1}}{\partial Y}\right)+\alpha A_{2}=0, \\
& i\left(\frac{\partial A_{2}}{\partial T}+\frac{p+2 n}{\sqrt{p^{2}+q^{2}}} \frac{\partial A_{2}}{\partial X}+\frac{q+2 m}{\sqrt{p^{2}+q^{2}}} \frac{\partial A_{2}}{\partial Y}\right)+\alpha A_{1}=0
\end{aligned}
$$


where $\alpha=\alpha_{n, m, 0}=\alpha_{-n,-m, 0}$. Coupled-mode equations (3.20)-(3.21) for two oblique waves cannot be reduced to the one-dimensional system (3.12)-(3.13), since the characteristics in the system (3.20)-(3.21) are no longer parallel.

The coupled-mode equations for three oblique resonant waves (2.18) can be derived similarly, subject to the resonance condition (2.19). Three characteristics along the wave vectors $\mathbf{k}_{1}=\mathbf{k}_{\mathrm{in}}, \mathbf{k}_{2}=\mathbf{k}_{\text {out }}^{\left(n_{1}, m_{1}, 0\right)}$, and $\mathbf{k}_{3}=\mathbf{k}_{\text {out }}^{\left(n_{2}, m_{2}, 0\right)}$ belong to the same $(X, Y)$-plane. The stationary transmission problem for the three oblique waves is hence a boundary-value problem on the $(X, Y)$-plane with three (linearly dependent) characteristic coordinates. Oblique interaction of three oblique resonant Bloch waves in a hexagonal crystal was considered numerically in [18].

4. Analysis of stationary transmission. The stationary transmission problem follows from separation of variables in the coupled-mode equations (3.9):

$$
A_{j}(\mathbf{X}, T)=a_{j}(\mathbf{X}) e^{-i \Omega T}, \quad j=1, \ldots, N,
$$

where $\Omega$ is the detuning frequency. When the boundary-value problem for $a_{j}(\mathbf{X})$ is well posed in a bounded domain, analytical solutions for the linear stationary coupledmode equations can be derived by using separation of variables and generalized Fourier series [24]. Exploiting these analytical solutions, integral invariants of the stationary transmission, reflection, and diffraction of the resonant Bloch waves can be computed explicitly. We analyze here the stationary coupled-mode equations for two and four counter-propagating and two oblique resonant Bloch waves.

4.1. Transmission of two counter-propagating waves. After separation of variables (4.1), the linear coupled-mode equations (3.12)-(3.13) reduce to the following ODE system:

$$
\begin{aligned}
i \frac{d a_{+}}{d Z}+\Omega a_{+}+\alpha a_{-} & =0, \\
-i \frac{d a_{-}}{d Z}+\alpha a_{+}+\Omega a_{-} & =0 .
\end{aligned}
$$

The problem (4.2)-(4.3) is defined on the interval $0 \leq Z \leq L_{Z}$. When the incident wave strikes the photonic crystal from the left, the linear system (4.2)-(4.3) is completed by the boundary conditions

$$
a_{+}(0)=\alpha_{+}, \quad a_{-}\left(L_{Z}\right)=0,
$$

where $\alpha_{+}$is the given amplitude of the incident wave at the left $(x, y)$-plane of the crystal. The general solution of the ODE system (4.2)-(4.3) is given explicitly as follows:

$$
\left(\begin{array}{c}
a_{+} \\
a_{-}
\end{array}\right)=c_{+}\left(\begin{array}{c}
\alpha \\
\Omega+i \kappa
\end{array}\right) e^{\kappa Z}+c_{-}\left(\begin{array}{c}
\alpha \\
\Omega-i \kappa
\end{array}\right) e^{-\kappa Z}
$$

where $c_{ \pm} \in \mathbb{C}$ are arbitrary and $\kappa \in \mathbb{C}$ is the root of the determinant equation

$$
\kappa=\sqrt{\alpha^{2}-\Omega^{2}} .
$$

When $\kappa=i K, K \in \mathbb{R}$, the linear dispersion relation $\Omega=\Omega(K)$ follows from the quadratic equation

$$
\Omega^{2}=\alpha^{2}+K^{2}
$$


The two branches of the dispersion relation (4.7) correspond to the two counterpropagating resonant waves. Their resonance leads to the photonic stop band, which is located in the interval $|\Omega|<|\alpha|$. Let $\Omega=0$ for simplicity; i.e., the detuning frequency is fixed in the middle of the stop band. The unique solution of the boundary-value problem (4.2)-(4.4) follows from the general solution (4.5):

$$
\left(\begin{array}{c}
a_{+} \\
a_{-}
\end{array}\right)=\frac{\alpha_{+}}{\cosh \alpha L_{Z}}\left(\begin{array}{c}
\cosh \alpha\left(L_{Z}-Z\right) \\
-i \sinh \alpha\left(L_{Z}-Z\right)
\end{array}\right) .
$$

The transmittance $T$ and reflectance $R$ are defined from the other boundary values of the solution (4.8),

$$
T=\left|\frac{a_{+}\left(L_{Z}\right)}{a_{+}(0)}\right|^{2}=\frac{1}{\cosh ^{2} \alpha L_{Z}}, \quad R=\left|\frac{a_{-}(0)}{a_{+}(0)}\right|^{2}=\frac{\sinh ^{2} \alpha L_{Z}}{\cosh ^{2} \alpha L_{Z}},
$$

such that the balance identity $T+R=1$ is satisfied. The analytical solution (4.8) for the two counter-propagating waves is well known [23] and is reproduced here for comparison with the case of four counter-propagating and two oblique waves on the plane.

4.2. Transmission of four counter-propagating waves. The stationary transmission of four counter-propagating waves in the coupled-mode equations (3.16)(3.19) is studied in the characteristic coordinates $(\xi, \eta)$ :

$$
\xi=\frac{X+Y}{2}, \quad \eta=\frac{X-Y}{2} .
$$

After the separation of variables (4.1), the linear coupled-mode equations (3.16)-(3.19) reduce to the $\mathrm{PDE}$ system

$$
\begin{array}{r}
i \frac{\partial a_{+}}{\partial \xi}+\Omega a_{+}+\alpha a_{-}+\beta\left(b_{+}+b_{-}\right)=0, \\
-i \frac{\partial a_{-}}{\partial \xi}+\alpha a_{+}+\Omega a_{-}+\beta\left(b_{+}+b_{-}\right)=0, \\
i \frac{\partial b_{+}}{\partial \eta}+\beta\left(a_{+}+a_{-}\right)+\Omega b_{+}+\alpha b_{-}=0, \\
-i \frac{\partial b_{-}}{\partial \eta}+\beta\left(a_{+}+a_{-}\right)+\alpha b_{+}+\Omega b_{-}=0 .
\end{array}
$$

The problem (4.11)-(4.14) is defined in a bounded domain on the plane $(\xi, \eta)$. We consider the rectangle

$$
\mathcal{D}=\left\{(\xi, \eta): 0 \leq \xi \leq L_{\xi}, 0 \leq \eta \leq L_{\eta}\right\},
$$

which corresponds to a rectangle in physical coordinates $(X, Y)$, rotated at $45^{\circ}$ in characteristic coordinates $(\xi, \eta)$. When the incident wave moves along the main diagonal in the $(X, Y)$-plane of the photonic crystal, the linear system (4.11)-(4.14) is completed by the boundary conditions

$$
a_{+}(0, \eta)=\alpha_{+}(\eta), \quad a_{-}\left(L_{\xi}, \eta\right)=0, \quad b_{+}(\xi, 0)=0, \quad b_{-}\left(\xi, L_{\eta}\right)=0,
$$

where $\alpha_{+}(\eta)$ is the given amplitude of the incident wave at the left boundary of the crystal. The linear dispersion relation $\Omega=\Omega\left(K_{\xi}, K_{\eta}\right)$, where $\left(K_{\xi}, K_{\eta}\right)$ are Fourier 
wave numbers, follows from the determinant equation of the linear PDE system (4.11)(4.14).

LEMma 4.1. The linear dispersion relation $\Omega=\Omega\left(K_{\xi}, K_{\eta}\right)$ is defined by the roots of $D\left(\Omega, K_{\xi}, K_{\eta}\right)$, where

$$
D\left(\Omega, K_{\xi}, K_{\eta}\right)=\left(\Omega^{2}-\alpha^{2}-K_{\xi}^{2}\right)\left(\Omega^{2}-\alpha^{2}-K_{\eta}^{2}\right)-4 \beta^{2}(\Omega-\alpha)^{2} .
$$

There exist real-valued roots of $D\left(0, K_{\xi}, K_{\eta}\right)=0$ for $\alpha^{2} \leq 4 \beta^{2}$, while no real-valued roots exist for $\alpha^{2}>4 \beta^{2}$.

Proof. The determinant equation follows from the PDE system (4.11)-(4.14) for the Fourier modes $e^{i\left(K_{\xi} \xi+K_{\eta} \eta\right)}$ in the explicit form

$$
D\left(\Omega, K_{\xi}, K_{\eta}\right)=\left|\begin{array}{cccc}
\Omega-K_{\xi} & \alpha & \beta & \beta \\
\alpha & \Omega+K_{\xi} & \beta & \beta \\
\beta & \beta & \Omega-K_{\eta} & \alpha \\
\beta & \beta & \alpha & \Omega+K_{\eta}
\end{array}\right| .
$$

Although the straightforward computations of $D\left(\Omega, K_{\xi}, K_{\eta}\right)$ are involved technically, it is easy to compute that

$$
\frac{\partial D}{\partial \Omega}=2 \Omega\left(\Omega^{2}-\alpha^{2}-K_{\xi}^{2}\right)+2 \Omega\left(\Omega^{2}-\alpha^{2}-K_{\eta}^{2}\right)-8 \beta^{2} \Omega+8 \alpha \beta^{2}
$$

and

$$
D\left(0, K_{\xi}, K_{\eta}\right)=\left(\alpha^{2}+K_{\xi}^{2}\right)\left(\alpha^{2}+K_{\eta}^{2}\right)-4 \alpha^{2} \beta^{2} .
$$

Integrating (4.19)-(4.20), we find that $D\left(\Omega, K_{\xi}, K_{\eta}\right)$ is given by (4.17). When $\alpha^{2}>$ $4 \beta^{2}$, the function $D\left(0, K_{\xi}, K_{\eta}\right)$ is positive definite on $\left(K_{\xi}, K_{\eta}\right) \in \mathbb{R}^{2}$ such that no real-valued roots $\left(K_{\xi}, K_{\eta}\right)$ exist for $\Omega=0$. When $\alpha^{2} \leq 4 \beta^{2}$, there exist two curves on the $\left(K_{\xi}, K_{\eta}\right)$-plane, which correspond to the real-valued roots of $D\left(0, K_{\xi}, K_{\eta}\right)$.

There are four surfaces of the dispersion relations $\Omega=\Omega\left(K_{\xi}, K_{\eta}\right)$, which correspond to the four resonant counter-propagating Bloch waves. When $\alpha^{2}>4 \beta^{2}$, the interaction of four resonant waves leads to a stop band near the zero detuning frequency $\Omega=0$. When $\alpha^{2} \leq 4 \beta^{2}$, no stop bands occur in the interaction of the four resonant waves. We consider solutions of the system (4.11)-(4.14) at $\Omega=0$. By separating variables [24], we reduce the PDE problem to two ODE problems as follows:

$$
\begin{aligned}
a_{+}(\xi, \eta) & =u_{+}(\xi) w_{a}(\eta), & & a_{-}(\xi, \eta)=u_{-}(\xi) w_{a}(\eta), \\
b_{+}(\xi, \eta) & =w_{b}(\xi) v_{+}(\eta), & & b_{-}(\xi, \eta)=w_{b}(\xi) v_{-}(\eta),
\end{aligned}
$$

where

$$
v_{+}(\eta)+v_{-}(\eta)=\mu w_{a}(\eta), \quad u_{+}(\xi)+u_{-}(\xi)=-\lambda w_{b}(\xi),
$$

parameters $(\lambda, \mu)$ are arbitrary, and vectors $\left(u_{+}, u_{-}\right)^{T}$ and $\left(v_{+}, v_{-}\right)^{T}$ solve the two uncoupled ODE systems

$$
\left(\begin{array}{cc}
i \partial_{\xi} & \alpha \\
\alpha & -i \partial_{\xi}
\end{array}\right)\left(\begin{array}{l}
u_{+} \\
u_{-}
\end{array}\right)=\beta \Gamma^{-1}\left(\begin{array}{cc}
1 & 1 \\
1 & 1
\end{array}\right)\left(\begin{array}{l}
u_{+} \\
u_{-}
\end{array}\right)
$$

and

$$
\left(\begin{array}{cc}
i \partial_{\eta} & \alpha \\
\alpha & -i \partial_{\eta}
\end{array}\right)\left(\begin{array}{l}
v_{+} \\
v_{-}
\end{array}\right)=\beta \Gamma\left(\begin{array}{ll}
1 & 1 \\
1 & 1
\end{array}\right)\left(\begin{array}{l}
v_{+} \\
v_{-}
\end{array}\right)
$$


where $\Gamma=\lambda / \mu$. The boundary conditions for (4.24)-(4.25) follow from (4.16) as follows:

$$
u_{+}(0)=1, \quad u_{-}\left(L_{\xi}\right)=0
$$

and

$$
v_{+}(0)=v_{-}\left(L_{\eta}\right)=0 .
$$

The homogeneous problem (4.25) and (4.27) defines the spectrum of $\Gamma$, while the inhomogeneous problem (4.24) and (4.26) defines a particular solution (4.21)-(4.22). The general solution of the problem (4.11)-(4.14) with the boundary values (4.16) is thought to be a linear superposition of infinitely many particular solutions, if the convergence and completeness of the decomposition formulas can be proved [24]. We first give solutions of the two problems above and then consider the orthogonality and completeness of the generalized Fourier series.

Lemma 4.2. All eigenvalues $\Gamma$ of the homogeneous problem (4.25) and (4.27) are given by nonzero roots of the characteristic equation

$$
\mathcal{R}=\left\{k \in \mathbb{C}:\left(\frac{k-\alpha}{k+\alpha}\right)^{2} e^{-2 i k L_{\eta}}=1, \operatorname{Re}(k) \geq 0, k \neq 0\right\}
$$

such that

$$
\Gamma=\frac{\alpha^{2}+k^{2}}{2 \alpha \beta} .
$$

Let $\alpha>0$. Then the roots $k \in \mathcal{R}$ are all located in the first open quadrant of $k \in \mathbb{C}$. Moreover, all roots are simple, and there exist $C>0$ and $N \in \mathbb{Z}_{+}$such that only one root $k \in \mathcal{R}$ is located in each rectangle:

$$
\mathcal{D}_{n}^{+}=\left\{k \in \mathbb{C}: \frac{\pi(4 n-1)}{2 L_{\eta}}<k<\frac{\pi(4 n+1)}{2 L_{\eta}}, 0<\operatorname{Im}(k)<C\right\}, \quad n \geq N,
$$

and

$$
\mathcal{D}_{n}^{-}=\left\{k \in \mathbb{C}: \frac{\pi(4 n+1)}{2 L_{\eta}}<k<\frac{\pi(4 n+3)}{2 L_{\eta}}, 0<\operatorname{Im}(k)<C\right\}, \quad n \geq N .
$$

Proof. The general solution of the ODE system (4.25) with the use of (4.29) is found explicitly as follows:

$$
\left(\begin{array}{c}
v_{+} \\
v_{-}
\end{array}\right)=c_{k}\left(\begin{array}{c}
\alpha-k \\
\alpha+k
\end{array}\right) e^{i k \eta}+c_{-k}\left(\begin{array}{c}
\alpha+k \\
\alpha-k
\end{array}\right) e^{-i k \eta}
$$

The coefficients $c_{k}$ and $c_{-k}$ satisfy the relations due to the boundary conditions (4.27):

$$
\frac{c_{k}}{c_{-k}}=\frac{k+\alpha}{k-\alpha}=\frac{k-\alpha}{k+\alpha} e^{-2 i k L_{\eta}},
$$

from which the characteristic equation (4.28) for roots $k \in \mathbb{C}$ follows. The symmetric roots $k$ and $(-k)$ correspond to the same $\Gamma$ and $v_{ \pm}(\eta)$. The root $k=0$ corresponds to the zero solution for $v_{ \pm}(\eta)$. Therefore, the roots $k=0$ and $\operatorname{Re}(k)<0$ are excluded 
from the definition of $\mathcal{R}$. The characteristic equation (4.28) results in the modulus equation

$$
\frac{|k-\alpha|}{|k+\alpha|}=\left|e^{i k L_{\eta}}\right|
$$

When $\alpha>0$, the left-hand side equals 1 at $\operatorname{Re}(k)=0$ and is smaller than 1 for $\operatorname{Re}(k)>0$. The right-hand side equals 1 at $\operatorname{Im}(k)=0$ and is larger than 1 for $\operatorname{Im}(k)<0$. Therefore, roots $k \in \mathcal{R}$ may occur only in the first open quadrant of $k \in \mathbb{C}$.

Let the roots $k \in \mathcal{R}$ be defined by the function $f(k)=(k-\alpha)^{2} e^{-2 i k L_{\eta}}-(k+\alpha)^{2}=$ 0 . Then,

$$
f^{\prime}(k)=-\frac{2 i(k+\alpha)}{(k-\alpha)}\left[\left(k^{2}-\alpha^{2}\right) L_{\eta}+2 i \alpha\right] .
$$

Since the values of $k^{2}-\alpha^{2}$ for $k \in \mathcal{R}$ are located in the upper half-plane of the complex plane, $f^{\prime}(k) \neq 0$ for $\alpha>0$ such that all roots of $k \in \mathcal{R}$ are simple.

The characteristic equation (4.28) splits into two sets of roots $\mathcal{R}_{+}$and $\mathcal{R}_{-}$such that $\mathcal{R}_{+} \cup \mathcal{R}_{-}=\mathcal{R}$, where

$$
\mathcal{R}_{ \pm}=\left\{k \in \mathbb{C}: \frac{k-\alpha}{k+\alpha} e^{-i k L_{\eta}}= \pm 1, \operatorname{Re}(k)>0\right\} .
$$

We consider the set $k \in \mathcal{R}_{+}$and rewrite it in the form $f(k)+g(k)=0$, where

$$
f(k)=e^{i k L_{\eta}}-1, \quad g(k)=\frac{2 \alpha}{k+\alpha} .
$$

The function $f(k)$ has a zero at

$$
k=k_{n}=\frac{2 \pi n}{L_{\eta}}, \quad n \geq 1 .
$$

Let us consider the domain $\tilde{\mathcal{D}}_{n}^{+}$:

$$
\tilde{\mathcal{D}}_{n}^{+}=\left\{k \in \mathbb{C}: \frac{\pi(4 n-1)}{2 L_{\eta}}<k<\frac{\pi(4 n+1)}{2 L_{\eta}},-C<\operatorname{Im}(k)<C\right\}, \quad n \geq N,
$$

for some large $C>0$ and $N \geq 1$, such that $\frac{\pi(4 n-1)}{2 L_{\eta}}>\alpha$. The domain $\tilde{\mathcal{D}}_{n}^{+}$surrounds a simple zero of $f(k)$ at $k=k_{n}$ such that $|f(k)|>|g(k)|$ on the boundary of $\tilde{\mathcal{D}}_{n}^{+}$. By Rouche's theorem, the function $f(k)+g(k)$ has the same number of zeros inside $\tilde{\mathcal{D}}_{n}^{+}$ as $f(k)$ does, i.e., only one zero. Since the roots are located in the first open quadrant of $k \in \mathbb{C}$, the root in $\tilde{\mathcal{D}}_{n}^{+}$is located in $\mathcal{D}_{n}^{+}$. The same analysis applies to the second set $k \in \mathcal{R}_{-}$in the domain $\mathcal{D}_{n}^{-}$.

Roots $k \in \mathcal{R}$ and $(-k) \in \mathcal{R}$ are shown in Figure 1 from the numerical solution of the characteristic equation (4.28) for $\alpha=1$ and $L_{\eta}=20$. In agreement with Lemma 4.2 , all roots $k \in \mathcal{R}$ are isolated points in the first open quadrant, which accumulate to the real axis of $k$ at infinity. The standard analysis of analytic functions at infinity leads to the asymptotic formula for distribution of large roots $k$ in the domain $|k|>k_{0} \gg 1$ :

$$
k_{n}^{+}=\frac{2 \pi n}{L_{\eta}}+\frac{i \alpha}{\pi n}+\mathrm{O}\left(\frac{1}{n^{2}}\right), \quad k_{n}^{-}=\frac{\pi(1+2 n)}{L_{\eta}}+\frac{2 i \alpha}{\pi(1+2 n)}+\mathrm{O}\left(\frac{1}{n^{2}}\right),
$$




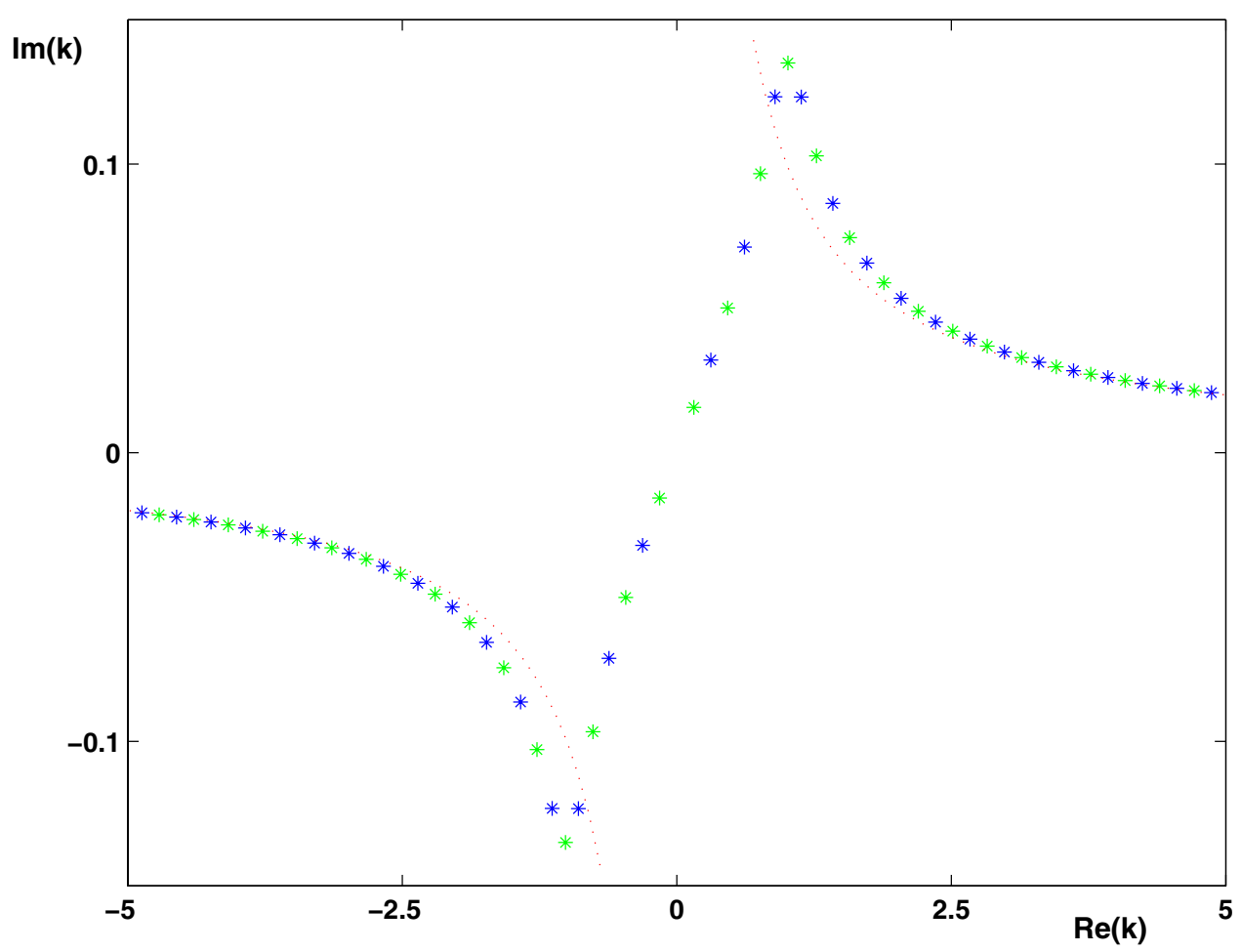

FIG. 1. Roots $k \in \mathcal{R}$ and $(-k) \in \mathcal{R}$ of the characteristic equation (4.28) for $\alpha=1$ and $L_{\eta}=20$. Dark dots show roots of $\mathcal{R}_{+}$, and bright dots show roots of $\mathcal{R}_{-}$. The dotted curves show the leading-order asymptotic approximation (4.36).

where $n$ is a large positive integer. The leading order of the asymptotic approximation (4.36) is also shown in Figure 1 by dotted curves. The two sets in (4.36) correspond to the splitting $k \in \mathcal{R}_{ \pm}$in (4.35). The eigenfunction $v(\eta)=v_{+}(\eta)+v_{-}(\eta)$ is symmetric (antisymmetric) with respect to $\eta=L_{\eta} / 2$ for $k \in \mathcal{R}_{+}\left(k \in \mathcal{R}_{-}\right)$. Moreover, explicit formulas for $v(\eta)$ follow from (4.32) and (4.33):

$$
\begin{aligned}
& k \in \mathcal{R}_{+}: v(\eta)=c_{+} \cos k\left(\frac{L_{\eta}}{2}-\eta\right), \\
& k \in \mathcal{R}_{-}: v(\eta)=c_{-} \sin k\left(\frac{L_{\eta}}{2}-\eta\right),
\end{aligned}
$$

where $\left(c_{+}, c_{-}\right)$are normalization constants. Asymptotic solutions (4.36) correspond to two sets of eigenfunctions

$$
\left\{\cos (\pi n \tilde{\eta}), \sin \left(\frac{\pi(2 n+1) \tilde{\eta}}{2}\right)\right\}, \quad \tilde{\eta}=\frac{2 \eta}{L_{\eta}}-1
$$

which solve the homogeneous Neumann problem on the normalized interval $-1 \leq \tilde{\eta} \leq$ 1 .

Lemma 4.3. Let $\Gamma$ be an eigenvalue of the problem (4.25) and (4.27). There exists a unique solution of the nonhomogeneous problem (4.24) and (4.26) for this $\Gamma$. 
Proof. A general solution of the ODE system (4.24) is found explicitly as follows:

$\left(\begin{array}{c}u_{+} \\ u_{-}\end{array}\right)=d_{k}\left(\begin{array}{c}\alpha^{2}+k^{2}-2 \beta^{2} \\ \lambda_{k}\left(\alpha^{2}+k^{2}\right)+2 \beta^{2}\end{array}\right) e^{i \alpha \lambda_{k} \eta}+d_{-k}\left(\begin{array}{c}\alpha^{2}+k^{2}-2 \beta^{2} \\ -\lambda_{k}\left(\alpha^{2}+k^{2}\right)+2 \beta^{2}\end{array}\right) e^{-i \alpha \lambda_{k} \eta}$, $(4.40)$

where

$$
\lambda_{k}=\sqrt{\frac{4 \beta^{2}}{\alpha^{2}+k^{2}}-1}
$$

The relation (4.41) satisfies the determinant equation (4.17) such that $D\left(0, \alpha \lambda_{k}, k\right)=$ 0 . Using the boundary conditions (4.26), coefficients $d_{k}$ and $d_{-k}$ are found uniquely, under the constraint

$$
u_{0}=\lambda_{k}\left(\alpha^{2}+k^{2}\right) \cos \alpha \lambda_{k} L_{\xi}+2 i \beta^{2} \sin \alpha \lambda_{k} L_{\xi} \neq 0 .
$$

We show that $u_{0} \neq 0$. The equation $u_{0}=0$ can be rewritten in the form

$$
\frac{\left(\lambda_{k}-1\right)^{2}}{\left(\lambda_{k}+1\right)^{2}}=e^{2 i \alpha \lambda_{k} L_{\xi}}
$$

By analysis of Lemma 4.2, it is clear that nonzero roots of the characteristic equation (4.43) may exist only in the first and third open quadrants of $\lambda_{k} \in \mathbb{C}$ for $\alpha>0$, such that the values of $\lambda_{k}^{2}+1$ are located in the upper half-plane of the complex plane. The zero root $\lambda_{k}=0$ is located on the real axis for $\lambda_{k}^{2}+1$. On the other hand, the values of $4 \beta^{2} /\left(\alpha^{2}+k^{2}\right)$ for $k \in \mathcal{R}$ are located in the lower half-plane. Therefore, the relation (4.41) leads to a contradiction, which proves that $u_{0} \neq 0$.

Solutions of the nonhomogeneous problem (4.24) and (4.26) with the normalization $u_{+}(0)=u_{0} \neq 0$ can be written explicitly by eliminating $d_{k}$ and $d_{-k}$ from the implicit form (4.40):

$$
\left(\begin{array}{l}
u_{+} \\
u_{-}
\end{array}\right)=\lambda_{k}\left(\alpha^{2}+k^{2}\right)\left(\begin{array}{l}
1 \\
0
\end{array}\right) \cos \alpha \lambda_{k}\left(L_{\xi}-\xi\right)+i\left(\begin{array}{c}
2 \beta^{2} \\
\alpha^{2}+k^{2}-2 \beta^{2}
\end{array}\right) \sin \alpha \lambda_{k}\left(L_{\xi}-\xi\right) .
$$

When the representation (4.21) is used for $\alpha_{+}(\eta)=a_{+}(0, \eta)$, the function $\alpha_{+}(\eta)$ is expanded as a series of scalar eigenfunctions $v(\eta)=v_{+}(\eta)+v_{-}(\eta)$, defined for roots $k \in \mathcal{R}$. This decomposition is possible only if the set of eigenfunctions $v(\eta)$ is orthogonal and complete.

LEMMA 4.4. There exists a set of normalized and orthogonal eigenfunctions $v_{j}(\eta)$ for distinct roots $k=k_{j} \in \mathcal{R}$, according to the inner product

$$
\int_{0}^{L_{\eta}} v_{i}(\eta) v_{j}(\eta) d \eta=\delta_{i, j}
$$

Proof. The set of adjoint eigenvectors to the problem (4.25) and (4.27) with respect to the standard inner product in $L^{2}\left(\left[0, L_{\eta}\right]\right)$ is given by the vectors $\left(\bar{v}_{-}, \bar{v}_{+}\right)^{T}$. As a result, the scalar eigenfunctions $v_{j}(\eta)$ for distinct roots $k=k_{j}$ satisfy the orthogonality relations (4.45) with $i \neq j$. The scalar eigenfunction $v(\eta)$ is found from (4.32) and (4.33) in the explicit form

$$
v(\eta)=c_{0}(k \cos k \eta+i \alpha \sin k \eta)
$$


where $c_{0}$ is a normalization constant. Integrating $v^{2}(\eta)$ on $\eta \in\left[0, L_{\eta}\right]$, we confirm that the eigenfunctions $v_{j}(\eta)$ can be normalized by the inner product (4.45) with $i=j$, under the constraint

$$
\left(k^{2}-\alpha^{2}\right) L_{\eta}+2 i \alpha \neq 0 .
$$

Since the roots $k \in \mathcal{R}$ are all simple, such that $f^{\prime}(k) \neq 0$ in (4.34), the constraint (4.47) is met.

Proposition 4.5. Any continuously differentiable complex-valued function $f(\eta)$ on $0 \leq \eta \leq L_{\eta}$ is uniquely represented by the series of eigenfunctions

$$
f(\eta)=\sum_{\text {all } k_{j} \in \mathcal{R}} c_{j} v_{j}(\eta), \quad c_{j}=\int_{0}^{L_{\eta}} f(\eta) v_{j}(\eta) d \eta,
$$

and the series converges to $f(\eta)$ uniformly on $0 \leq \eta \leq L_{\eta}$.

Proof. It follows from (4.25) and (4.27) that the scalar eigenfunction $v(\eta)$ solves the second-order boundary-value problem

$$
v^{\prime \prime}+k^{2} v=0
$$

such that

$$
i v^{\prime}(0)+\alpha v(0)=0, \quad-i v^{\prime}\left(L_{\eta}\right)+\alpha v\left(L_{\eta}\right)=0 .
$$

The Sommerfeld radiation boundary conditions (4.50) explain why the spectrum of the formally self-adjoint operator (4.49) is complex-valued. The statement of the proposition follows from the expansion theorem [7, p. 303], since the theorem's condition is satisfied: $A_{2,4}=1$, where $A_{2,4}$ is the determinant of the second and fourth columns of the matrix $A$, associated with the boundary conditions

$$
A=\left(\begin{array}{cccc}
\alpha & i & 0 & 0 \\
0 & 0 & \alpha & -i
\end{array}\right) \text {. }
$$

As a result, the Fourier series of asymptotic eigenfunctions (4.39) approximates the series expansion (4.48) for large roots $k=k_{n}^{ \pm}$uniformly on $\eta \in\left[0, L_{\eta}\right]$. The uniform convergence of (4.48) follows from that of the Fourier series [24].

Using separation of variables and convergence of series of eigenfunctions, we summarize the existence and uniqueness results on the generalized Fourier series solutions of the linear boundary-value problem (4.11)-(4.14) and (4.16) with $\Omega=0$.

Proposition 4.6. Let the set $\left\{c_{j}\right\}$ be uniquely defined by the series (4.48) for $f(\eta)=\alpha_{+}(\eta)$. There exists a unique solution of the boundary-value problem (4.11)(4.14) and (4.16) with $\Omega=0$ in the domain (4.15):

$$
\begin{aligned}
& a_{+}(\xi, \eta)=\sum_{\text {all } k_{j} \in \mathcal{R}} c_{j} \frac{u_{+j}(\xi)}{u_{+j}(0)}\left(v_{+j}(\eta)+v_{-j}(\eta)\right), \\
& a_{-}(\xi, \eta)=\sum_{\text {all } k_{j} \in \mathcal{R}} c_{j} \frac{u_{-j}(\xi)}{u_{+j}(0)}\left(v_{+j}(\eta)+v_{-j}(\eta)\right), \\
& b_{+}(\xi, \eta)=-\sum_{\text {all } k_{j} \in \mathcal{R}} c_{j} \frac{u_{+j}(\xi)+u_{-j}(\xi)}{\Gamma_{j} u_{+j}(0)} v_{+j}(\eta), \\
& b_{-}(\xi, \eta)=-\sum_{\text {all } k_{j} \in \mathcal{R}} c_{j} \frac{u_{+j}(\xi)+u_{-j}(\xi)}{\Gamma_{j} u_{+j}(0)} v_{-j}(\eta) .
\end{aligned}
$$


We illustrate the generalized Fourier series solutions (4.51)-(4.54) with two examples: (i) a single term of the generalized Fourier series and (ii) a constant input function $\alpha_{+}(\eta)=\alpha_{+}$. For both examples, we compute the integral invariants for the incident $\left(\mathcal{I}_{\text {in }}\right)$, transmitted $\left(\mathcal{I}_{\text {out }}\right)$, reflected $\left(\mathcal{I}_{\text {ref }}\right)$, and diffracted $\left(\mathcal{I}_{\text {dif }}\right)$ waves from their definitions:

$$
\begin{array}{rlrl}
\mathcal{I}_{\text {in }} & =\int_{0}^{L_{\eta}}\left|a_{+}(0, \eta)\right|^{2} d \eta, & \mathcal{I}_{\text {out }}=\int_{0}^{L_{\eta}}\left|a_{+}\left(L_{\xi}, \eta\right)\right|^{2} d \eta, \\
\mathcal{I}_{\text {ref }}=\int_{0}^{L_{\eta}}\left|a_{-}(0, \eta)\right|^{2} d \eta, & \mathcal{I}_{\text {dif }}=\int_{0}^{L_{\xi}}\left(\left|b_{+}\left(\xi, L_{\eta}\right)\right|^{2}+\left|b_{-}(\xi, 0)\right|^{2}\right) d \xi .
\end{array}
$$

Let the transmittance $T$, reflectance $R$, and diffractance $D$ be defined from the relations

$$
T=\frac{\mathcal{I}_{\text {out }}}{\mathcal{I}_{\text {in }}}, \quad R=\frac{\mathcal{I}_{\text {ref }}}{\mathcal{I}_{\text {in }}}, \quad D=\frac{\mathcal{I}_{\text {dif }}}{\mathcal{I}_{\text {in }}} .
$$

The integral invariants satisfy the balance identity

$$
R+T+D=1,
$$

which follows from integration of the balance equation

$$
\frac{\partial}{\partial \xi}\left(\left|a_{+}\right|^{2}-\left|a_{-}\right|^{2}\right)+\frac{\partial}{\partial \eta}\left(\left|b_{+}\right|^{2}-\left|b_{-}\right|^{2}\right)=0 .
$$

First, we consider a single term of the Fourier series solutions (4.51)-(4.54). The transmittance and reflectance for $k \in \mathcal{R}$ are found from (4.44) in the explicit form

$$
\begin{aligned}
T_{k} & =\left|\frac{\lambda_{k}\left(\alpha^{2}+k^{2}\right)}{\lambda_{k}\left(\alpha^{2}+k^{2}\right) \cos \alpha \lambda_{k} L_{\xi}+2 i \beta^{2} \sin \alpha \lambda_{k} L_{\xi}}\right|^{2}, \\
R_{k} & =\left|\frac{\left(\alpha^{2}+k^{2}-2 \beta^{2}\right) \sin \alpha \lambda_{k} L_{\xi}}{\lambda_{k}\left(\alpha^{2}+k^{2}\right) \cos \alpha \lambda_{k} L_{\xi}+2 i \beta^{2} \sin \alpha \lambda_{k} L_{\xi}}\right|^{2},
\end{aligned}
$$

while the diffractance is found from the balance identity as $D_{k}=1-T_{k}-R_{k}$. These integral invariants of the stationary transmission for $\alpha=1$ and $L_{\xi}=L_{\eta}=20$ are shown in Figure 2 for $\beta=0.25$ and in Figure 3 for $\beta=0.75$. In the first case, when $\alpha^{2}>4 \beta^{2}$, there is a stop band at $\Omega=0$, such that all modes are fully reflected except for small losses due to diffraction. In the second case, when $\alpha^{2}<4 \beta^{2}$, there is no stop band at $\Omega=0$, such that transmittance and diffractance are large for smaller values of $|k|$ and become negligible for larger values of $|k|$.

Next, we consider a constant input function:

$$
\alpha_{+}(\eta)=\alpha_{+}, \quad \eta \in\left[0, L_{\eta}\right],
$$

when $c_{j}$ can be found from (4.48),

$$
c_{j}=\frac{4 i \alpha \alpha_{+}}{k_{j}\left[L\left(k_{j}^{2}-\alpha^{2}\right)+2 i \alpha\right]}, \quad k_{j} \in \mathcal{R}_{+},
$$

and $c_{j}=0$ for $k_{j} \in \mathcal{R}_{-}$. The solution surfaces $\left|a_{ \pm}(\xi, \eta)\right|^{2}$ and $\left|b_{ \pm}(\xi, \eta)\right|^{2}$ in the domain (4.15) are shown for $\alpha=1, L_{\xi}=L_{\eta}=20$, and $\alpha_{+}=1$ in Figure 4 for $\beta=0.25$ and 


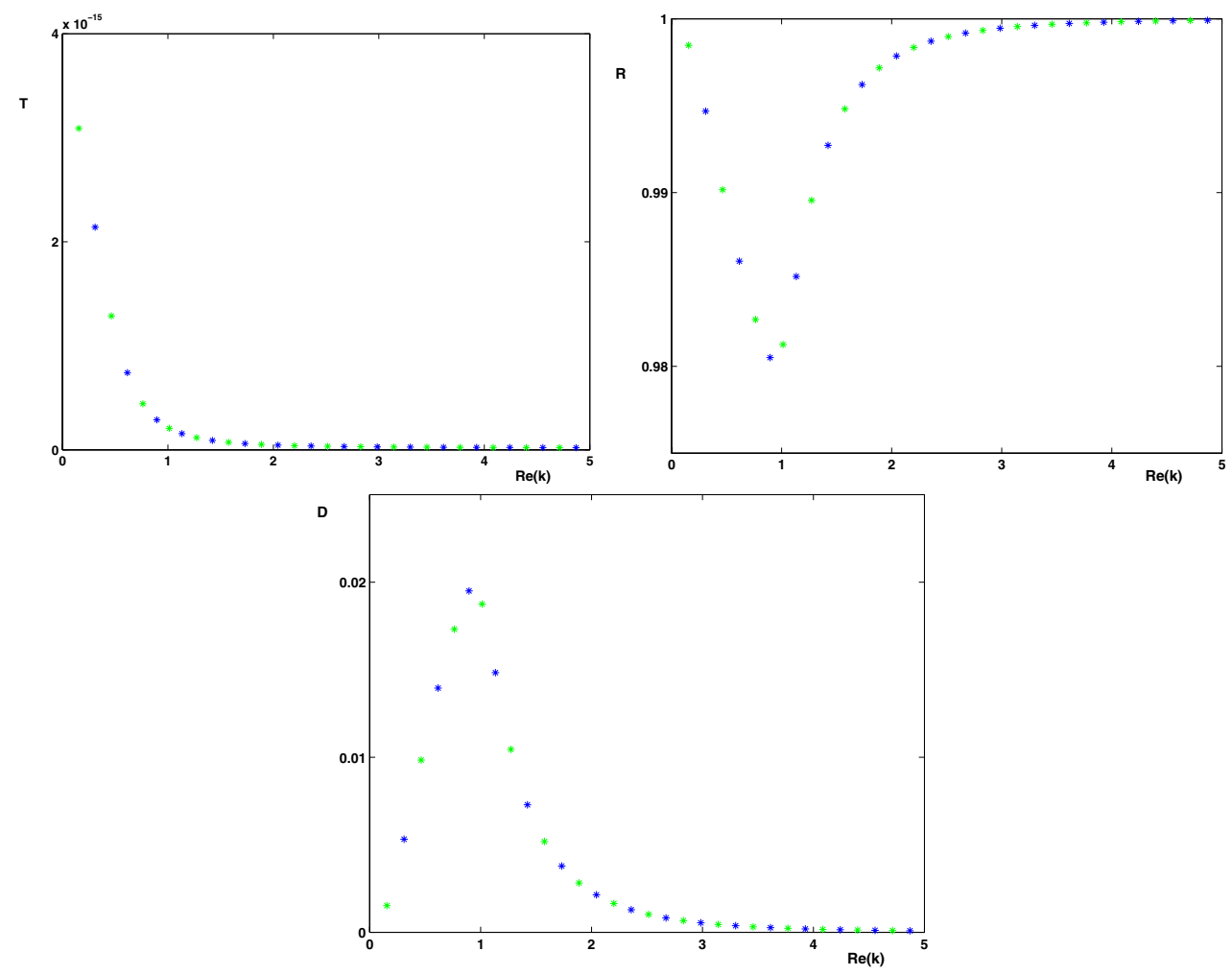

FIG. 2. Transmittance $\left(T_{k}\right)$, reflectance $\left(R_{k}\right)$, and diffractance $\left(D_{k}\right)$ versus $\operatorname{Re}(k)$ for the roots $k \in \mathcal{R}$ when $\alpha=1, \beta=0.25$, and $L_{\xi}=L_{\eta}=20$.

in Figure 5 for $\beta=0.75$. We can see from the figures that the boundary conditions (4.16) are satisfied by the truncated generalized Fourier series (4.51)-(4.54) with only 30 first terms.

The Parseval identity cannot be applied to eigenfunctions $v_{j}(\eta)$, because the inner product $(4.45)$ is not the standard inner product in $L^{2}\left(\left[0, L_{\eta}\right]\right)$. As a result, the energy spectrum of $I_{\text {out }}, I_{\text {ref }}$, and $I_{\text {dif }}$ cannot be decomposed into a superposition of the squared amplitudes $\left|c_{j}\right|^{2}$. Nevertheless, the numerical values for $T, R$, and $D$ can be found from numerical integration of the solution surfaces (4.55)-(4.56). The numerical values are

$$
\begin{aligned}
& \beta=0.25: T \approx 3 \times 10^{-15}, \quad R \approx 0.9853, \quad D \approx 0.0147, \\
& \beta=0.75: T \approx 0.7394, \quad R \approx 0.0133, \quad D \approx 0.2473,
\end{aligned}
$$

such that $T+R+D \approx 1$. When $\alpha^{2}>4 \beta^{2}$, there exists a stop band at $\Omega=0$, and the incident wave is reflected from the photonic crystal with energy loss of $1.5 \%$ due to diffraction. When $\alpha^{2}<4 \beta^{2}$, there is no stop band at $\Omega=0$, and the incident wave is transmitted along the photonic crystal with energy loss of $26 \%$ due to reflection and diffraction.

4.3. Transmission of two oblique waves. The stationary transmission of two oblique waves in the coupled-mode equations (3.20)-(3.21) becomes diagonal in the 

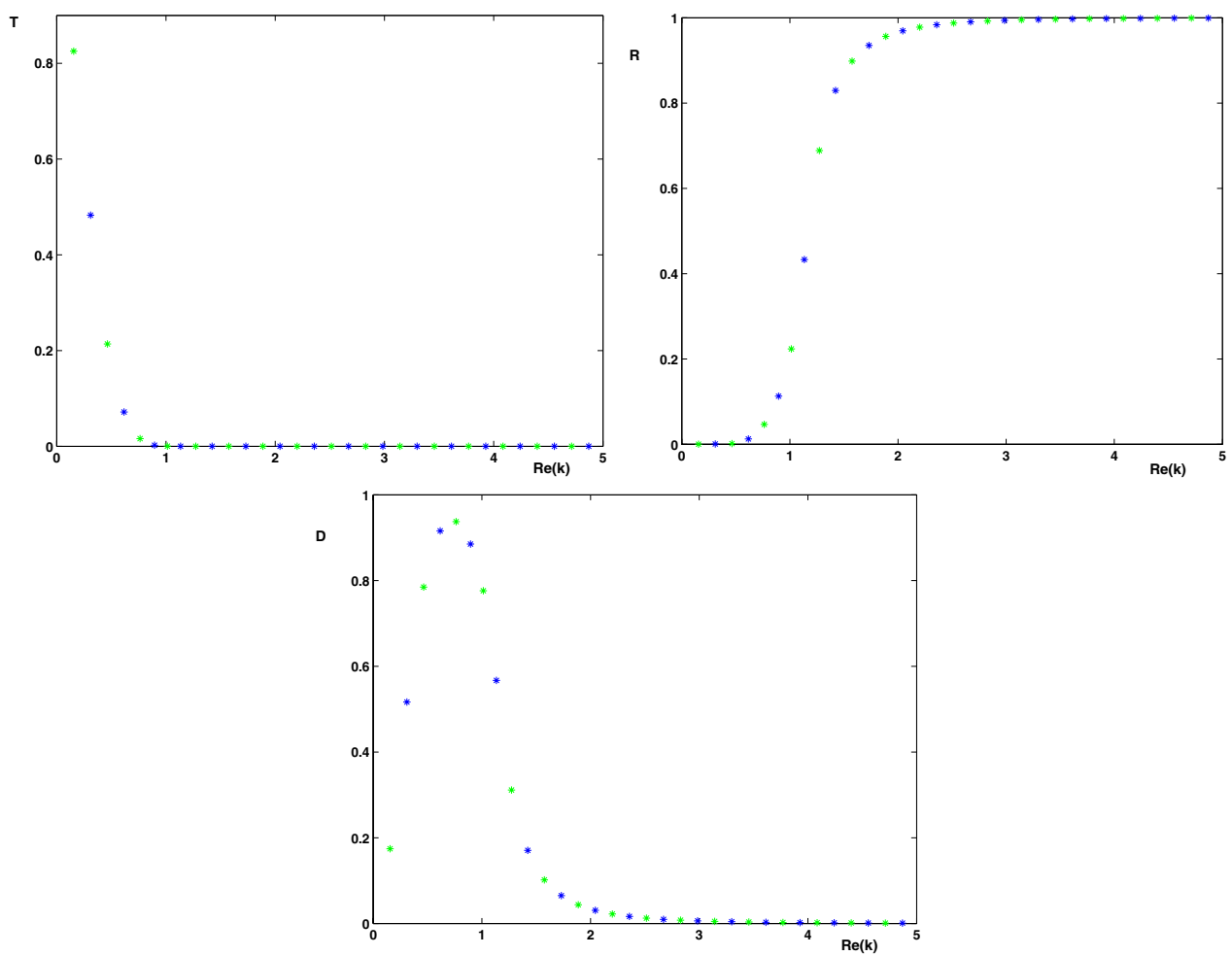

FIG. 3. Transmittance $\left(T_{k}\right)$, reflectance $\left(R_{k}\right)$, and diffractance $\left(D_{k}\right)$ versus $\operatorname{Re}(k)$ for the roots $k \in \mathcal{R}$ when $\alpha=1, \beta=0.75$, and $L_{\xi}=L_{\eta}=20$.

characteristic coordinates $(\xi, \eta)$ :

$$
X=\frac{p \xi+(p+2 n) \eta}{\sqrt{p^{2}+q^{2}}}, \quad Y=\frac{q \xi+(q+2 m) \eta}{\sqrt{p^{2}+q^{2}}}
$$

After the separation of variables (4.1), the linear coupled-mode equations (3.20)-(3.21) reduce to the PDE system

$$
\begin{aligned}
& i \frac{\partial a_{1}}{\partial \xi}+\Omega a_{1}+\alpha a_{2}=0 \\
& i \frac{\partial a_{2}}{\partial \eta}+\alpha a_{1}+\Omega a_{2}=0
\end{aligned}
$$

Coordinate axes $(\xi, \eta)$ are parallel to the wave vectors $\mathbf{k}_{1}=\mathbf{k}_{\text {in }}$ and $\mathbf{k}_{2}=\mathbf{k}_{\text {out }}^{(n, m, 0)}$, but they are no longer orthogonal. The problem (4.65)-(4.66) is defined in a bounded domain on the plane $(\xi, \eta)$. We consider the same rectangle $\mathcal{D}$, defined by (4.15). When the incident wave is illuminated in the direction of the wave vector $\mathbf{k}_{1}$ but not in the direction of the wave vector $\mathbf{k}_{2}$, the linear system (4.65)-(4.66) is completed by the boundary conditions

$$
a_{1}(0, \eta)=\alpha_{1}(\eta), \quad a_{2}(\xi, 0)=0
$$



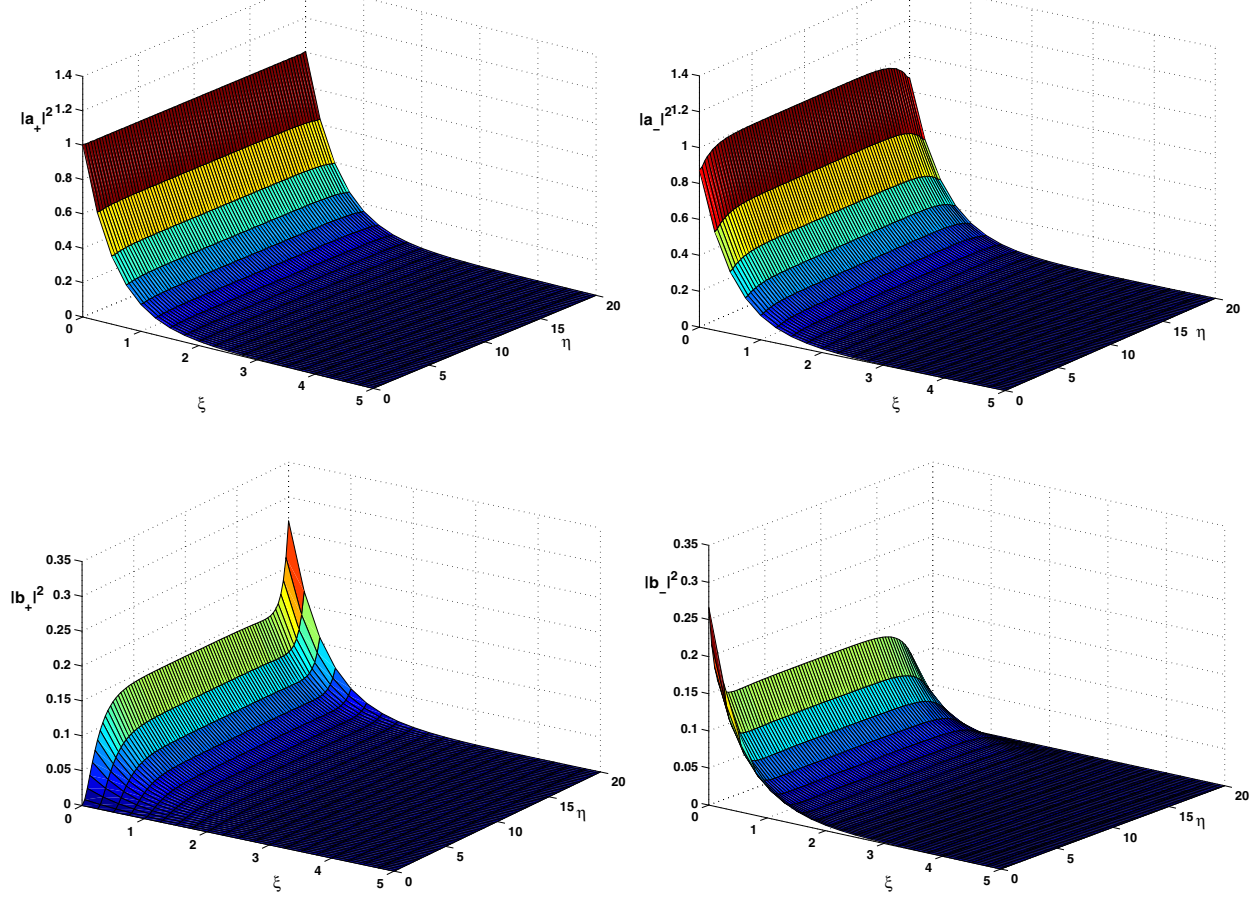

FIG. 4. Solution surfaces $\left|a_{ \pm}\right|^{2}(\xi, \eta)$ and $\left|b_{ \pm}\right|^{2}(\xi, \eta)$ on the domain (4.15) for $\alpha=1, \beta=0.25$, $L_{\xi}=L_{\eta}=20$, and $\alpha_{+}=1$.

The linear dispersion relation $\Omega=\Omega\left(K_{\xi}, K_{\eta}\right)$, where $\left(K_{\xi}, K_{\eta}\right)$ are Fourier wave numbers, is given explicitly as

$$
\left(\Omega-\frac{K_{\xi}+K_{\eta}}{2}\right)^{2}=\alpha^{2}+\left(\frac{K_{\xi}-K_{\eta}}{2}\right)^{2}
$$

Two surfaces of the dispersion relation (4.68) correspond to the two oblique resonant waves. In a moving reference frame on the plane $(\xi, \eta)$ there exists a stop band in the dispersion relation (4.68). We consider solutions of the system (4.65)-(4.66) at $\Omega=0$ by using the Fourier transform

$$
\begin{aligned}
& a_{1}(\xi, \eta)=\int_{-\infty}^{\infty} k c(k) e^{i \alpha\left(k^{-1} \xi+k \eta\right)} d k \\
& a_{2}(\xi, \eta)=\int_{-\infty}^{\infty} c(k) e^{i \alpha\left(k^{-1} \xi+k \eta\right)} d k
\end{aligned}
$$

It follows from the boundary conditions (4.67) that

$$
k c(k)=\frac{\alpha}{2 \pi} \int_{0}^{L_{\eta}} \alpha_{1}(\eta) e^{-i \alpha k \eta} d \eta, \quad k \in \mathbb{R},
$$

and

$$
0=\int_{-\infty}^{\infty} c(k) e^{i \alpha k^{-1} \xi} d k, \quad 0 \leq \xi \leq L_{\xi}
$$



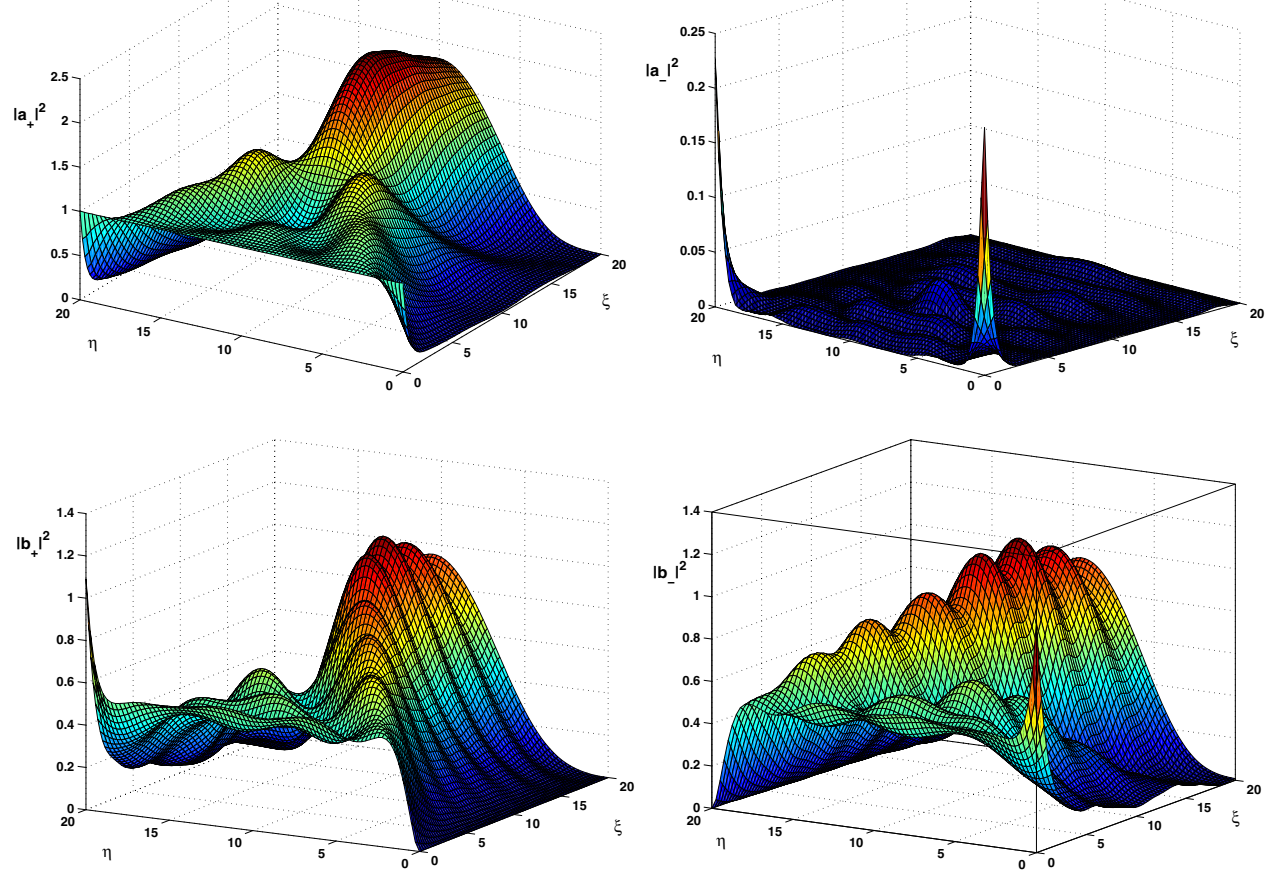

FIG. 5. Solution surfaces $\left|a_{ \pm}\right|^{2}(\xi, \eta)$ and $\left|b_{ \pm}\right|^{2}(\xi, \eta)$ on the domain (4.15) for $\alpha=1, \beta=0.75$, $L_{\xi}=L_{\eta}=20$, and $\alpha_{+}=1$.

Interchanging integrals, we reduce the constraint (4.72) to the form

$$
0=\frac{\alpha}{2 \pi i} \int_{0}^{L_{\eta}} \alpha_{1}(\eta)\left(\int_{-\infty}^{\infty} \frac{\sin \alpha\left(k \eta-k^{-1} \xi\right)}{k} d k\right) d \eta, \quad 0 \leq \xi \leq L_{\xi} .
$$

The inner integral is zero for $\xi>0$ and $\eta>0$, due to the table integral 3.871 on p. 474 of [9]. Therefore, the constraint (4.72) is satisfied, and a unique solution of the problem (4.65)-(4.67) exists in the form (4.69)-(4.71).

We illustrate the Fourier transform solution (4.69)-(4.70) with the constant input function

$$
\alpha_{1}(\eta)=\alpha_{1}, \quad \eta \in\left[0, L_{\eta}\right]
$$

when $c(k)$ can be found from (4.71):

$$
c(k)=\frac{\alpha_{1}}{2 \pi i} \frac{1-e^{-i \alpha k L_{\eta}}}{k^{2}}, \quad k \in \mathbb{R} .
$$

Evaluating Fourier integrals (4.69)-(4.70) with the help of the table integral 3.871 on p. 474 of [9], we find the explicit solution of the stationary problem:

$$
a_{1}(\xi, \eta)=\alpha_{1} J_{0}(2 \alpha \sqrt{\xi \eta}), \quad a_{2}(\xi, \eta)=\frac{i \alpha_{1} \sqrt{\eta}}{\sqrt{\xi}} J_{1}(2 \alpha \sqrt{\xi \eta})
$$



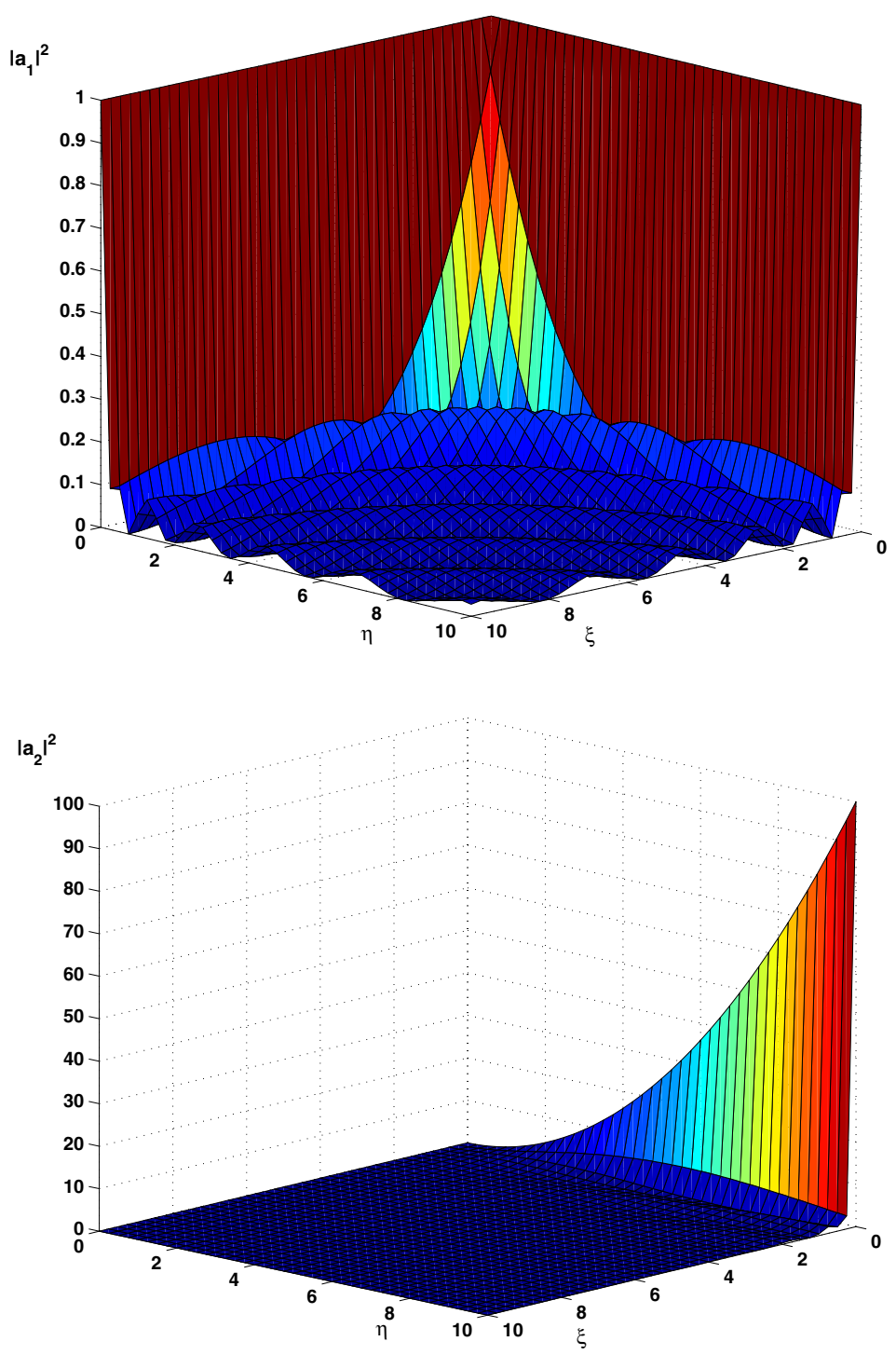

FIG. 6. Solution surfaces $\left|a_{1}\right|^{2}(\xi, \eta)$ and $\left|a_{2}\right|^{2}(\xi, \eta)$ on the domain (4.15) for $\alpha=1, L_{\xi}=L_{\eta}=$ 10 , and $\alpha_{1}=1$.

where $J_{0,1}(z)$ are Bessel functions [9]. Figure 6 shows the solution surfaces $\left|a_{1}(\xi, \eta)\right|^{2}$ and $\left|a_{2}(\xi, \eta)\right|^{2}$ in the domain (4.15) for $\alpha=1, L_{\xi}=L_{\eta}=10$, and $\alpha_{1}=1$. The integral invariants for the stationary transmission follow from integration of the balance equation:

$$
\frac{\partial}{\partial \xi}\left|a_{1}\right|^{2}+\frac{\partial}{\partial \eta}\left|a_{2}\right|^{2}=0
$$


We define the incident $\left(\mathcal{I}_{\text {in }}\right)$, transmitted $\left(\mathcal{I}_{\text {out }}\right)$, and diffracted $\left(\mathcal{I}_{\text {dif }}\right)$ intensities by

$$
\mathcal{I}_{\text {in }}=\int_{0}^{L_{\eta}}\left|a_{1}(0, \eta)\right|^{2} d \eta, \quad \mathcal{I}_{\text {out }}=\int_{0}^{L_{\eta}}\left|a_{1}\left(L_{\xi}, \eta\right)\right|^{2} d \eta, \quad \mathcal{I}_{\text {dif }}=\int_{0}^{L_{\xi}}\left|a_{2}\left(\xi, L_{\eta}\right)\right|^{2} d \xi .
$$

The transmittance $(T)$ and diffractance $(D)$ are defined by the same relations (4.57), and the balance identity $T+D=1$ follows from integration of the balance equation (4.77). The numerical values for $T$ and $D$ are found from numerical integration of the solution surfaces (4.78) as follows:

$$
T \approx 0.032, \quad D \approx 0.968
$$

such that $T+D \approx 1$. These values show that the incident wave is diffracted to the oblique resonance wave such that only $3.2 \%$ of the wave energy remains in the transmitted wave.

4.4. General transmission problems. A general system of coupled-mode equations (3.9) can be diagonalized in characteristic coordinates, similarly to the case of four counter-propagating and two oblique resonant waves. The characteristic coordinates are introduced from the set of resonant wave vectors as follows:

$$
\frac{\partial}{\partial \xi_{j}}=\frac{\mathbf{k}_{j, x}}{k} \frac{\partial}{\partial X}+\frac{\mathbf{k}_{j, y}}{k} \frac{\partial}{\partial Y}+\frac{\mathbf{k}_{j, z}}{k} \frac{\partial}{\partial Z}, \quad j=1, \ldots, N
$$

such that the characteristic coordinate $\xi_{j}$ extends in the direction of the wave vector $\mathbf{k}_{j}$. The characteristic coordinates $\left(\xi_{1}, \ldots, \xi_{N}\right) \in \mathbb{R}^{N}$ are related to the physical coordinates $(X, Y, Z) \in \mathbb{R}^{3}$ as follows:

$$
\mathbf{X}=\mathbf{X}_{0}+\sum_{j=1}^{N} \xi_{j} \frac{\mathbf{k}_{j}}{k},
$$

where $\mathbf{X}_{0} \in \mathbb{R}^{3}$ is an arbitrary point. The boundary-value problem for the linear stationary transmission with $\Omega=0$ can be rewritten in the form

$$
i \frac{\partial a_{j}}{\partial \xi_{j}}+\sum_{k \neq j} \hat{\alpha}_{j, k} a_{k}=0, \quad j=1, \ldots, N .
$$

We consider the domain of definition in the cone $\left(\xi_{1}, \ldots, \xi_{N}\right) \in \mathbb{R}_{+}^{N}$, subject to the Goursat boundary values

$$
a_{j}\left(\xi_{1}, \ldots, \xi_{j-1}, 0, \xi_{j+1}, \ldots, \xi_{N}\right)=\alpha_{j}\left(\xi_{1}, \ldots, \xi_{j-1}, \xi_{j+1}, \ldots, \xi_{N}\right), \quad j=1, \ldots, N .
$$

The Goursat boundary-value problem (4.81)-(4.82) can be rewritten as the Volterra integral equations:

$$
a_{j}\left(\xi_{j}\right)=\alpha_{j}+i \int_{0}^{\xi_{j}} \sum_{k \neq j} \hat{\alpha}_{j, k} a_{k}\left(\xi_{j}^{\prime}\right) d \xi_{j}^{\prime} .
$$

By the contraction mapping principle [11], there exists a unique solution of the Volterra equations (4.83) in the cone $\left(\xi_{1}, \ldots, \xi_{N}\right) \in \mathbb{R}_{+}^{N}$, such that we have the following result. 
TheOREM 4.7. Let $\mathcal{D}$ be a convex domain in $\mathbb{R}^{3}$, which is cut by the characteristic coordinate projections $\xi_{j}=0, j=1, \ldots, N$. There exists a unique solution $a_{j}=$ $a_{j}\left(\xi_{1}, \ldots, \xi_{N}\right)$, which corresponds to the boundary-value problem (4.81)-(4.82) and depends smoothly on the boundary values $\alpha_{j}, j=1, \ldots, N$.

If $N=\operatorname{rank}\left(\mathbf{k}_{1}, \ldots, \mathbf{k}_{N}\right)$, there exists only one domain $\mathcal{D} \subset \mathbb{R}^{N}$, which corresponds to the cone $\xi_{j} \geq 0, j=1, \ldots, N$. The case of two oblique waves on the plane $(X, Y)$ gives an example of this situation for $N=2$. The Goursat problem (4.81)(4.82) is rewritten as the PDE problem (4.65)-(4.66) with the boundary values (4.67), which is solved with the explicit Fourier transform solutions (4.69)-(4.71).

If $N>\operatorname{rank}\left(\mathbf{k}_{1}, \ldots, \mathbf{k}_{N}\right)$, the characteristic coordinates $\left(\xi_{1}, \ldots, \xi_{N}\right)$ are linearly dependent such that there exist multiple ways to choose a convex domain $\mathcal{D} \subset \mathbb{R}^{3}$ that corresponds to the cone $\xi_{j} \geq 0, j=1, \ldots, N$. The case of four counterpropagating waves on the plane $(X, Y)$ gives an example of this situation for $N=4$ and $\operatorname{rank}\left(\mathbf{k}_{1}, \ldots, \mathbf{k}_{N}\right)=2$. In this case, we have chosen that $\xi_{1}=\xi, \xi_{2}=\eta$, $\xi_{3}=L_{\eta}-\eta$, and $\xi_{4}=L_{\xi}-\xi$, such that $0 \leq \xi \leq L_{\xi}$ and $0 \leq \eta \leq L_{\eta}$. As a result, the Goursat problem (4.81)-(4.82) is rewritten as the PDE problem (4.11)-(4.14) with the boundary values (4.16). Theorem 4.7 does not guarantee well-posedness of (4.11)-(4.14), while explicit Fourier series solutions (4.51)-(4.54) do (see Proposition $4.6)$.

5. Summary and open problems. We have shown that the coupled-mode equations can be used for analysis and modeling of resonant interaction of Bloch waves in low-contrast cubic-lattice three-dimensional photonic crystals. The analytical solutions for the linear stationary transmission problem are found by using separation of variables and generalized Fourier series. We have proved that the linear stationary boundary-value problem is well-posed in the context of four counter-propagating and two oblique waves on the plane. We have also given general results on well-posedness of the general linear stationary transmission problem.

It remains an open problem to prove well-posedness of the nonlinear stationary boundary-value problem for small-norm and finite-norm solutions. Nonstationary transmission problems are also of interest, and very few analytical results are available on local and global well-posedness of the nonstationary nonlinear coupled-mode equations. Finally, numerical approximations of the stationary and nonstationary, fully nonlinear coupled-mode equations can be constructed in bounded domains with the method of orthogonal polynomials [17]. All these problems are beyond the scope of the present work.

Appendix A. Nonlinear coupled-mode equations with cubic nonlinearities. Modeling of nonlinear photonic band-gap crystals with cubic (Kerr) nonlinearities is based on the Maxwell equations, where the polarization vector depends nonlinearly on the electric field vector $\mathbf{E}$ (see [13]). When the nonlinearity terms are small, nonlocal (dispersive) terms in the polarization vector can be neglected, and the low-contrast weakly nonlinear photonic crystals can be modeled with the Maxwell equations (1.1), where the refractive index $n=n\left(\mathbf{x},|\mathbf{E}|^{2}\right)$ is decomposed into the linear and nonlinear parts [23]:

$$
n\left(\mathbf{x},|\mathbf{E}|^{2}\right)=n_{0}+\epsilon n_{1}(\mathbf{x})+\epsilon n_{2}(\mathbf{x})|\mathbf{E}|^{2},
$$

where $n_{0}$ is constant and $\epsilon$ is of small parameter. When the photonic crystal has cubic-lattice structure, the periodic functions $n_{1}(\mathbf{x})$ and $n_{2}(\mathbf{x})$ are expanded into the 
triple Fourier series (3.4) and

$$
n_{2}(\mathbf{x})=n_{0} \sum_{(n, m, l) \in \mathbb{Z}^{3}} \beta_{n, m, l} e^{i k_{0}(n x+m y+l z)}, \quad \beta_{n, m, l}=\bar{\beta}_{-n,-m,-l},
$$

where the factor $n_{0}$ is included for convenience. Derivation of the nonlinear coupledmode equations is based on rigorous methods of Lyapunov-Schmidt reductions [16]. Equivalently, the formal derivation can be recovered with perturbation series expansions [19], which follows the formalism (3.2) and (3.3) outlined in section 3. The first-order correction term $\mathbf{E}_{1}(\mathbf{x}, t)$ solves the nonhomogeneous problem (3.8) with additional nonlinear terms:

$$
\begin{aligned}
\nabla^{2} \mathbf{E}_{1}-\frac{n_{0}^{2}}{c^{2}} \frac{\partial^{2} \mathbf{E}_{1}}{\partial t^{2}}=2 & \frac{n_{0}^{2} \omega}{c^{2}} \frac{\partial^{2} \mathbf{E}_{0}}{\partial T \partial t}-2 k\left(\nabla \cdot \nabla_{X}\right) \mathbf{E}_{0} \\
& +\frac{2 n_{0} n_{1}(\mathbf{x})}{c^{2}} \frac{\partial^{2} \mathbf{E}_{0}}{\partial t^{2}}+\frac{2}{n_{0}} \nabla\left(\nabla n_{1} \cdot \mathbf{E}_{0}\right) \\
& +\frac{2 n_{0} n_{2}(\mathbf{x})}{c^{2}}\left|\mathbf{E}_{0}\right|^{2} \frac{\partial^{2} \mathbf{E}_{0}}{\partial t^{2}}+\frac{2}{n_{0}} \nabla\left(\nabla n_{2}\left|\mathbf{E}_{0}\right|^{2} \cdot \mathbf{E}_{0}\right) .
\end{aligned}
$$

The cubic nonlinear terms generate $N^{3}$ terms from the leading-order solution (3.3), which all give resonant terms by means of the triple series (A.2). By removing the resonant terms, the nonlinear coupled-mode equations for $A_{j}(\mathbf{X}, T), j=1, \ldots, N$, are derived in the general form:

$$
i\left(\frac{\partial A_{j}}{\partial T}+\left(\frac{\mathbf{k}_{j}}{k} \cdot \nabla_{X}\right) A_{j}\right)+\sum_{k \neq j} \hat{\alpha}_{j, k} A_{k}+\sum_{1 \leq k_{1}, k_{2}, k_{3} \leq N} \hat{\beta}_{j, k_{1}, k_{2}, k_{3}} A_{k_{1}} A_{k_{2}} \bar{A}_{k_{3}}=0
$$

where the elements $\left\{\hat{\beta}_{j, k_{1}, k_{2}, k_{3}}\right\}_{1 \leq j, k_{1}, k_{2}, k_{3} \leq N}$ are related to the Fourier coefficients of the resonant waves $\left\{\beta_{n, m, l}\right\}_{(n, m, l) \in \mathcal{S}}$. The explicit forms of the nonlinear coupledmode equations are given below for two and four counter-propagating and two oblique resonant Bloch waves.

The nonlinear coupled-mode equations for two counter-propagating waves (3.10) generalize the linear equations (3.12)-(3.13) as follows:

$$
\begin{aligned}
& i\left(\frac{\partial A_{+}}{\partial T}+\frac{\partial A_{+}}{\partial Z}\right)+\alpha A_{-}+\beta_{0,0,0}\left(\left|A_{+}\right|^{2}+2\left|A_{-}\right|^{2}\right) A_{+} \\
& \begin{aligned}
(\text { A. } 5) \quad & \beta_{0,0,1}\left(2\left|A_{+}\right|^{2}+\left|A_{-}\right|^{2}\right) A_{-}+\beta_{0,0,-1} A_{+}^{2} \bar{A}_{-}+\beta_{0,0,2} \bar{A}_{+} A_{-}^{2}=0, \\
i\left(\frac{\partial A_{-}}{\partial T}-\frac{\partial A_{-}}{\partial Z}\right) & +\alpha A_{+}+\beta_{0,0,0}\left(2\left|A_{+}\right|^{2}+\left|A_{-}\right|^{2}\right) A_{-} \\
& +\beta_{0,0,-1}\left(\left|A_{+}\right|^{2}+2\left|A_{-}\right|^{2}\right) A_{+}+\beta_{0,0,1} \bar{A}_{+} A_{-}^{2}+\beta_{0,0,-2} A_{+}^{2} \bar{A}_{-}=0 .
\end{aligned}
\end{aligned}
$$

The system (A.5)-(A.6) is reviewed in $[8,23]$ for $\beta_{0,0,1}=\beta_{0,0,2}=0$ and analyzed in $[14,15]$ for $\beta_{0,0,1} \neq 0$ and $\beta_{0,0,2}=0$. When $\beta_{0,0,1}, \beta_{0,0,2} \neq 0$, the system (3.12)(3.13) is the most general coupled-mode system for Bragg resonance of two counterpropagating waves $[6,22]$.

The nonlinear coupled-mode equations for four counter-propagating waves (3.14) generalize the linear equations (3.16)-(3.19) as follows:

$$
i\left(\frac{\partial A_{+}}{\partial T}+\frac{\partial A_{+}}{\partial X}+\frac{\partial A_{+}}{\partial Y}\right)+\alpha A_{-}+\beta\left(B_{+}+B_{-}\right)+F_{+}\left(A_{+}, A_{-}, B_{+}, B_{-}\right)=0
$$


(A.8) $i\left(\frac{\partial A_{-}}{\partial T}-\frac{\partial A_{-}}{\partial X}-\frac{\partial A_{-}}{\partial Y}\right)+\alpha A_{+}+\beta\left(B_{+}+B_{-}\right)+F_{-}\left(A_{+}, A_{-}, B_{+}, B_{-}\right)=0$

(A.9) $i\left(\frac{\partial B_{+}}{\partial T}+\frac{\partial B_{+}}{\partial X}-\frac{\partial B_{+}}{\partial Y}\right)+\beta\left(A_{+}+A_{-}\right)+\alpha B_{-}+G_{+}\left(A_{+}, A_{-}, B_{+}, B_{-}\right)=0$,

(A.10) $i\left(\frac{\partial B_{-}}{\partial T}-\frac{\partial B_{-}}{\partial X}+\frac{\partial B_{-}}{\partial Y}\right)+\beta\left(A_{+}+A_{-}\right)+\alpha B_{+}+G_{-}\left(A_{+}, A_{-}, B_{+}, B_{-}\right)=0$,

where the cubic nonlinear functions are given by

$$
\begin{aligned}
& F_{+}=\beta_{0,0,0}\left(\left(\left|A_{+}\right|^{2}+2\left|A_{-}\right|^{2}+2\left|B_{+}\right|^{2}+2\left|B_{-}\right|^{2}\right) A_{+}+2 \bar{A}_{-} B_{+} B_{-}\right) \\
& +\beta_{0,-1,0}\left(A_{+}^{2} \bar{B}_{+}+2 A_{+} \bar{A}_{-} B_{-}\right)+\beta_{-1,0,0}\left(A_{+}^{2} \bar{B}_{-}+2 A_{+} \bar{A}_{-} B_{+}\right) \\
& +\beta_{1,1,0}\left(\left(2\left|A_{+}\right|^{2}+\left|A_{-}\right|^{2}+2\left|B_{+}\right|^{2}+2\left|B_{-}\right|^{2}\right) A_{-}+2 \bar{A}_{+} B_{+} B_{-}\right) \\
& +\beta_{0,1,0}\left(\left(2\left|A_{+}\right|^{2}+2\left|A_{-}\right|^{2}+\left|B_{+}\right|^{2}+2\left|B_{-}\right|^{2}\right) B_{+}+2 A_{+} A_{-} \bar{B}_{-}\right) \\
& +\beta_{-1,1,0}\left(2 A_{+} B_{+} \bar{B}_{-}+\bar{A}_{-} B_{+}^{2}\right)+\beta_{1,-1,0}\left(2 A_{+} \bar{B}_{+} B_{-}+\bar{A}_{-} B_{-}^{2}\right) \\
& +\beta_{1,0,0}\left(\left(2\left|A_{+}\right|^{2}+2\left|A_{-}\right|^{2}+2\left|B_{+}\right|^{2}+\left|B_{-}\right|^{2}\right) B_{-}+2 A_{+} A_{-} \bar{B}_{+}\right) \\
& +\beta_{2,0,0}\left(\bar{A}_{+} B_{-}^{2}+2 A_{-} \bar{B}_{+} B_{-}\right)+\beta_{2,1,0}\left(2 \bar{A}_{+} A_{-} B_{-}+A_{-}^{2} \bar{B}_{+}\right) \\
& +\beta_{1,2,0}\left(A_{-}^{2} \bar{B}_{-}+2 \bar{A}_{+} A_{-} B_{+}\right)+\beta_{2,-1,0} \bar{B}_{+} B_{-}^{2}+\beta_{-1,2,0} B_{+}^{2} \bar{B}_{-} \\
& +\beta_{0,2,0}\left(\bar{A}_{+} B_{+}^{2}+2 A_{-} B_{+} \bar{B}_{-}\right)+\beta_{-1,-1,0} A_{+}^{2} \bar{A}_{-}+\beta_{2,2,0} \bar{A}_{+} A_{-}^{2}, \\
& F_{-}=\beta_{-1,-1,0}\left(\left(\left|A_{+}\right|^{2}+2\left|A_{-}\right|^{2}+2\left|B_{+}\right|^{2}+2\left|B_{-}\right|^{2}\right) A_{+}+2 \bar{A}_{-} B_{+} B_{-}\right) \\
& +\beta_{-1,-2,0}\left(A_{+}^{2} \bar{B}_{+}+2 A_{+} \bar{A}_{-} B_{-}\right)+\beta_{-2,-1,0}\left(A_{+}^{2} \bar{B}_{-}+2 A_{+} \bar{A}_{-} B_{+}\right) \\
& +\beta_{0,0,0}\left(\left(2\left|A_{+}\right|^{2}+\left|A_{-}\right|^{2}+2\left|B_{+}\right|^{2}+2\left|B_{-}\right|^{2}\right) A_{-}+2 \bar{A}_{+} B_{+} B_{-}\right) \\
& +\beta_{-1,0,0}\left(\left(2\left|A_{+}\right|^{2}+2\left|A_{-}\right|^{2}+\left|B_{+}\right|^{2}+2\left|B_{-}\right|^{2}\right) B_{+}+2 A_{+} A_{-} \bar{B}_{-}\right) \\
& +\beta_{-2,0,0}\left(2 A_{+} B_{+} \bar{B}_{-}+\bar{A}_{-} B_{+}^{2}\right)+\beta_{0,-2,0}\left(2 A_{+} \bar{B}_{+} B_{-}+\bar{A}_{-} B_{-}^{2}\right) \\
& +\beta_{0,-1,0}\left(\left(2\left|A_{+}\right|^{2}+2\left|A_{-}\right|^{2}+2\left|B_{+}\right|^{2}+\left|B_{-}\right|^{2}\right) B_{-}+2 A_{+} A_{-} \bar{B}_{+}\right) \\
& +\beta_{1,-1,0}\left(\bar{A}_{+} B_{-}^{2}+2 A_{-} \bar{B}_{+} B_{-}\right)+\beta_{1,0,0}\left(2 \bar{A}_{+} A_{-} B_{-}+A_{-}^{2} \bar{B}_{+}\right) \\
& +\beta_{0,1,0}\left(A_{-}^{2} \bar{B}_{-}+2 \bar{A}_{+} A_{-} B_{+}\right)+\beta_{1,-2,0} \bar{B}_{+} B_{-}^{2}+\beta_{-2,1,0} B_{+}^{2} \bar{B}_{-} \\
& +\beta_{-1,1,0}\left(\bar{A}_{+} B_{+}^{2}+2 A_{-} B_{+} \bar{B}_{-}\right)+\beta_{-2,-2,0} A_{+}^{2} \bar{A}_{-}+\beta_{1,1,0} \bar{A}_{+} A_{-}^{2}, \\
& G_{+}=\beta_{0,-1,0}\left(\left(\left|A_{+}\right|^{2}+2\left|A_{-}\right|^{2}+2\left|B_{+}\right|^{2}+2\left|B_{-}\right|^{2}\right) A_{+}+2 \bar{A}_{-} B_{+} B_{-}\right) \\
& +\beta_{0,-2,0}\left(A_{+}^{2} \bar{B}_{+}+2 A_{+} \bar{A}_{-} B_{-}\right)+\beta_{-1,-1,0}\left(A_{+}^{2} \bar{B}_{-}+2 A_{+} \bar{A}_{-} B_{+}\right) \\
& +\beta_{1,0,0}\left(\left(2\left|A_{+}\right|^{2}+\left|A_{-}\right|^{2}+2\left|B_{+}\right|^{2}+2\left|B_{-}\right|^{2}\right) A_{-}+2 \bar{A}_{+} B_{+} B_{-}\right) \\
& +\beta_{0,0,0}\left(\left(2\left|A_{+}\right|^{2}+2\left|A_{-}\right|^{2}+\left|B_{+}\right|^{2}+2\left|B_{-}\right|^{2}\right) B_{+}+2 A_{+} A_{-} \bar{B}_{-}\right) \\
& +\beta_{-1,0,0}\left(2 A_{+} B_{+} \bar{B}_{-}+\bar{A}_{-} B_{+}^{2}\right)+\beta_{1,-2,0}\left(2 A_{+} \bar{B}_{+} B_{-}+\bar{A}_{-} B_{-}^{2}\right) \\
& +\beta_{1,-1,0}\left(\left(2\left|A_{+}\right|^{2}+2\left|A_{-}\right|^{2}+2\left|B_{+}\right|^{2}+\left|B_{-}\right|^{2}\right) B_{-}+2 A_{+} A_{-} \bar{B}_{+}\right) \\
& +\beta_{2,-1,0}\left(\bar{A}_{+} B_{-}^{2}+2 A_{-} \bar{B}_{+} B_{-}\right)+\beta_{2,0,0}\left(2 \bar{A}_{+} A_{-} B_{-}+A_{-}^{2} \bar{B}_{+}\right) \\
& +\beta_{1,1,0}\left(A_{-}^{2} \bar{B}_{-}+2 \bar{A}_{+} A_{-} B_{+}\right)+\beta_{2,-2,0} \bar{B}_{+} B_{-}^{2}+\beta_{-1,1,0} B_{+}^{2} \bar{B}_{-} \\
& +\beta_{0,1,0}\left(\bar{A}_{+} B_{+}^{2}+2 A_{-} B_{+} \bar{B}_{-}\right)+\beta_{-1,-2,0} A_{+}^{2} \bar{A}_{-}+\beta_{2,1,0} \bar{A}_{+} A_{-}^{2}, \\
& G_{-}=\beta_{-1,0,0}\left(\left(\left|A_{+}\right|^{2}+2\left|A_{-}\right|^{2}+2\left|B_{+}\right|^{2}+2\left|B_{-}\right|^{2}\right) A_{+}+2 \bar{A}_{-} B_{+} B_{-}\right) \\
& +\beta_{-1,-1,0}\left(A_{+}^{2} \bar{B}_{+}+2 A_{+} \bar{A}_{-} B_{-}\right)+\beta_{-2,0,0}\left(A_{+}^{2} \bar{B}_{-}+2 A_{+} \bar{A}_{-} B_{+}\right) \\
& +\beta_{0,1,0}\left(\left(2\left|A_{+}\right|^{2}+\left|A_{-}\right|^{2}+2\left|B_{+}\right|^{2}+2\left|B_{-}\right|^{2}\right) A_{-}+2 \bar{A}_{+} B_{+} B_{-}\right) \\
& +\beta_{-1,1,0}\left(\left(2\left|A_{+}\right|^{2}+2\left|A_{-}\right|^{2}+\left|B_{+}\right|^{2}+2\left|B_{-}\right|^{2}\right) B_{+}+2 A_{+} A_{-} \bar{B}_{-}\right) \\
& +\beta_{-2,1,0}\left(2 A_{+} B_{+} \bar{B}_{-}+\bar{A}_{-} B_{+}^{2}\right)+\beta_{0,-1,0}\left(2 A_{+} \bar{B}_{+} B_{-}+\bar{A}_{-} B_{-}^{2}\right) \\
& +\beta_{0,0,0}\left(\left(2\left|A_{+}\right|^{2}+2\left|A_{-}\right|^{2}+2\left|B_{+}\right|^{2}+\left|B_{-}\right|^{2}\right) B_{-}+2 A_{+} A_{-} \bar{B}_{+}\right)
\end{aligned}
$$




$$
\begin{aligned}
& +\beta_{1,0,0}\left(\bar{A}_{+} B_{-}^{2}+2 A_{-} \bar{B}_{+} B_{-}\right)+\beta_{1,1,0}\left(2 \bar{A}_{+} A_{-} B_{-}+A_{-}^{2} \bar{B}_{+}\right) \\
& +\beta_{0,2,0}\left(A_{-}^{2} \bar{B}_{-}+2 \bar{A}_{+} A_{-} B_{+}\right)+\beta_{1,-1,0} \bar{B}_{+} B_{-}^{2}+\beta_{-2,2,0} B_{+}^{2} \bar{B}_{-} \\
& +\beta_{-1,2,0}\left(\bar{A}_{+} B_{+}^{2}+2 A_{-} B_{+} \bar{B}_{-}\right)+\beta_{-2,-1,0} A_{+}^{2} \bar{A}_{-}+\beta_{1,2,0} \bar{A}_{+} A_{-}^{2} .
\end{aligned}
$$

The nonlinear coupled-mode equations for two oblique waves (2.16) generalize the linear equations (3.20)-(3.21) as follows:

$$
\begin{gathered}
i\left(\frac{\partial A_{1}}{\partial T}+\frac{p}{\sqrt{p^{2}+q^{2}}} \frac{\partial A_{1}}{\partial X}+\frac{q}{\sqrt{p^{2}+q^{2}}} \frac{\partial A_{1}}{\partial Y}\right)+\alpha A_{2}+\beta_{0,0,0}\left(\left|A_{1}\right|^{2}+2\left|A_{2}\right|^{2}\right) A_{1} \\
\begin{array}{r}
\text { (A. } 11) \quad+\beta_{-n,-m, 0}\left(2\left|A_{1}\right|^{2}+\left|A_{2}\right|^{2}\right) A_{2}+\beta_{n, m, 0} A_{1}^{2} \bar{A}_{2}+\beta_{-2 n,-2 m, 0} \bar{A}_{1} A_{2}^{2}=0, \\
i\left(\frac{\partial A_{2}}{\partial T}+\frac{p+2 n}{\sqrt{p^{2}+q^{2}}} \frac{\partial A_{2}}{\partial X}+\frac{q+2 m}{\sqrt{p^{2}+q^{2}}} \frac{\partial A_{2}}{\partial Y}\right)+\alpha A_{1}+\beta_{0,0,0}\left(2\left|A_{1}\right|^{2}+\left|A_{2}\right|^{2}\right) A_{1} \\
\quad+\beta_{n, m, 0}\left(\left|A_{1}\right|^{2}+2\left|A_{2}\right|^{2}\right) A_{1}+\beta_{-n,-m, 0} \bar{A}_{1} A_{2}^{2}+\beta_{2 n, 2 m, 0} A_{1}^{2} \bar{A}_{2}=0 .
\end{array} \\
\begin{array}{r}
\text { (A.12) } \quad
\end{array}
\end{gathered}
$$

The system (A.11)-(A.12) and its generalization to three oblique resonant waves are reviewed in $[18,19]$.

Acknowledgments. The authors thank Walter Craig, Ted Sargent, and Jamin Sheriff for collaboration and useful discussions. This paper was completed during the visit of D. P. to Universidad Autonoma del Estado de Mexico, organized by Dr. M. Aguero Granados and supported by research grant SEPPROMEP Mexico /103.5/03/309.

\section{REFERENCES}

[1] N. Akozbek And S. John, Optical solitary waves in two- and three-dimensional nonlinear photonic band-gap structures, Phys. Rev. E, 57 (1998), pp. 2287-2319.

[2] N. Akozber and S. John, Self-induced transparency solitary waves in a doped nonlinear photonic band gap material, Phys. Rev. E, 58 (1998), pp. 3876-3895.

[3] A. Arraf And C.M. De Sterke, Coupled-mode equations for quadratically nonlinear deep gratings, Phys. Rev. E, 58 (1998), pp. 7951-7958.

[4] A. Babin AND A. Figotin, Nonlinear photonic crystals: I. Quadratic nonlinearity, Waves Random Media, 11 (2001), pp. R31-R102.

[5] A. Babin AND A. Figotin, Nonlinear photonic crystals: II. Interaction classification for quadratic nonlinearities, Waves Random Media, 12 (2002), pp. R25-R52.

[6] N. Bhat And J.E. SiPe, Optical pulse propagation in nonlinear photonic crystals, Phys. Rev. E, 64 (2001), paper 056604.

[7] E.A. Coddington and N. Levinson, Theory of Ordinary Differential Equations, McGrawHill, New York, 1955.

[8] R.H. Goodman, M.I. Weinstein, and P.J. Holmes, Nonlinear propagation of light in onedimensional periodic structures, J. Nonlinear Sci., 11 (2001), pp. 123-168.

[9] I.S. Gradshtein And I.M. Ryzhik, Table of Integrals, Series, and Products, Academic Press, New York, 2000.

[10] C. Kittel, Introduction to Solid-State Physics, John Wiley \& Sons, New York, 1996.

[11] A.N. Kolmogorov And S.V. Fomin, Elements of the Theory of Functions and Functional Analysis, Nauka, Moscow, 1989.

[12] P. Kuchment, Floquet Theory for Partial Differential Operators, Birkhäuser, Basel, 1993.

[13] P. Kuchment, The mathematics of photonic crystals, in Mathematical Modeling in Optical Sciences, G. Bao, L. Cowsar, and W. Masters, eds., Frontiers in Appl. Math 22, SIAM, Philadelphia, 1999, pp. 207-272.

[14] D. Pelinovsky, J. Sears, L. Brzozowski, and E.H. Sargent, Stable all-optical limiting in nonlinear periodic structures. I: Analysis, J. Opt. Soc. Amer. B Opt. Phys., 19 (2002), pp. $43-53$.

[15] D.E. Pelinovsky And A. Scheel, Spectral analysis of stationary light transmission in nonlinear photonic structures, J. Nonlinear Sci., 13 (2003), pp. 347-396. 
[16] G. SCHNEIDER AND H. UECKER, Existence and stability of modulating pulse solutions in Maxwell's equations describing nonlinear optics, Z. Angew. Math. Phys., 54 (2003), pp. $677-712$.

[17] J. SHEN, Efficient spectral-Galerkin method I: Direct solvers of second-and fourth-order equations using Legendre polynomials, SIAM J. Sci. Comput., 15 (1994), pp. 1489-1505.

[18] J.L. SherifF, I.A. Goldthorpe, ANd E.H. SArgent, Optical limiting and intensity-dependent diffraction from low-contrast nonlinear periodic media: Coupled-mode analysis, Phys. Rev. E, 70 (2004), paper 036616.

[19] J.L. ShERIFF, Coupled-mode theory in low-contrast nonlinear photonic crystals, B.Sc. thesis, ECE Department, University of Toronto, Toronto, ON, 2003.

[20] C.M. de Sterke and J.E. Sipe, Envelope-function approach for the electrodynamics of nonlinear periodic structures, Phys. Rev. A, 38 (1988), pp. 5149-5165.

[21] C.M. De Sterke And J.E. Sipe, Extensions and generalizations of an envelope-function approach for the electrodynamics of nonlinear periodic structures, Phys. Rev. A, 39 (1989), pp. 5163-5178.

[22] C.M. De Sterke, D.G. Salinas, and J.E. Sipe, Coupled-mode theory for light propagation through deep nonlinear gratings, Phys. Rev. E, 54 (1996), pp. 1969-1989.

[23] C.M. De Sterke And J.E. Sipe, Gap solitons, Progress in Optics, 33 (1994), pp. 203-260.

[24] W. Strauss, Partial Differential Equations: An Introduction, John Wiley \& Sons, New York, 1992. 